\title{
A Study of Variation of Mixed Hodge Structure
}

\author{
By \\ Masaki KaSHIWARA*
}

§. Imintoduction

In $[\mathrm{S}-\mathbb{Z}]$, Steenbrink and Zucker proposed and studied "admissible variation of Mixed Hodge structure" on a curve as an "appropriate" notion. Here, we give a definition of admissible variation of mixed Hodge structure on a complex variety by "curve test" and afford the evidence that this is a good notion.

D.1. We shall recall what is an admissible VMHS on a curve, proposed by Steenbrink-Zucker. Let $X$ be a curve and $X^{*}$ a Zariski open subset of $X$. A graded polarizable variation of mixed Hodge structure on $X^{*}$ is called admissible, if it satisfies

(a) There exists the relative monodromy filtration of the logarithm of the unipotent part of the monodromy around each point in $X \backslash X^{*}$ with respect to the weight filtration.

(b) The Hodge filtration $F$ extends to a subsheaf of the canonical extension such that $\mathrm{Gr}^{W} \mathrm{Gr}_{F}$ is locally free. Furthermore for any morphism $f: D \rightarrow X$ from unit disc $D$ to $X$ the Hodge filtration of $f^{*} H$ satisfies the same conditions.

D.2. Let $X$ be a complex analytic space, and $X^{*}$ a non singular Zariski open subset of $X$. A graded polarizable VMHS on $X$ is called admissible (on $X$ ) if its restriction to any curve in $X$ (not totally contained in $X \backslash X^{*}$ ) is admissible. Such a definition by "curve test" is seldom a good notion unless it is a property of codimension 1 (or 0,2 ). This means that if this property is true outside a closed analytic subset of codimension 2 (or 1,3 ) then this holds on the whole

Received June 4, 1986.

* Research Institute for Mathematical Sciences, Kyoto University, Kyoto 606, Japan. 
space. As an example we recall the regularity of integrable connections or the quasi-unipotency of constructible sheaves.

In this article, we prove that "admissible" VMHS has a property of codimension 1 when $X$ is non-singular and $X^{*}$ is the complement of normally crossing hypersurface.

The proof makes an essential use of the purity theorem for a variation of Hodge structure.

This paper includes also a study of general notions which will be useful for further studies of mixed Hodge structures.

The author thanks Y. Shimizu for helpful discussions.

\section{$\S 1$. Admissible Variation of Mixed Hodge Structure}

1.1. We shall recall the definition of Hodge structure. For an integer $w$, a Hodge structure $\mathrm{H}$ of weight $w$ is a triplet $\left(\mathrm{H}_{C}, F(\mathrm{H}), \bar{F}(\mathrm{H})\right)$, where $\mathrm{H}_{C}$ is a finite-dimensional $C$-vector space and $F(H), \bar{F}(\mathrm{H})$ are two finite filtrations of $\mathrm{H}_{C}$ such that $\mathrm{H}_{C} \simeq \underset{p+q=w}{\oplus} \mathrm{H}^{p q}(\mathrm{H})$. Here $\mathrm{H}^{p q}(\mathrm{H})=F^{p}(\mathrm{H}) \cap \bar{F}^{q}(\mathrm{H})$. Let $\mathrm{C}(\mathrm{H})$ be the Weil operator defined by $\left.\mathrm{C}(\mathrm{H})\right|_{H^{p q}(\mathrm{H})} ^{p}=i^{p-q}$.

1.2. For a $C$-vector space $V$, let us denote by $\bar{V}$ the complex conjugate and let $-: V \rightarrow \bar{V}$ be the $\mathbb{R}$-linear isomorphism such that $\overline{a v}=\bar{a} \bar{v}$ for $a \in \mathbb{C}$ and $v \in V$. For a homomorphism $f: V \rightarrow V^{\prime}$ of $\mathbb{C}$-vector space let $\bar{f}: \bar{V} \rightarrow \bar{V}^{\prime}$ be the homomorphism given by $\bar{f}(\bar{v})=\overline{f(v)}$.

For a Hodge structure $\mathrm{H}$ of weight $w$, the complex conjugate $\overline{\mathrm{H}}$ of $\mathrm{H}$ is the Hodge structure of weight $w$ defined by $(\overline{\mathrm{H}})_{C}=\left(\mathrm{H}_{C}\right)^{-}, F^{p}(\overline{\mathrm{H}})=\bar{F}^{p}(\mathrm{H})^{-}$, $\bar{F}^{q}(\overline{\mathrm{H}})=F^{q}(\mathrm{H})^{-}$. We have $\mathrm{H}^{p q}(\overline{\mathrm{H}})=\mathrm{H}^{q p}(\mathrm{H})^{-}$and $\mathrm{C}(\overline{\mathrm{H}})=\mathrm{C}(\mathrm{H})^{-}$

1.3. A polarization of a Hodge structure $\mathrm{H}$ of weight $w$ is a bilinear homomorphism $S: \mathrm{H}_{\boldsymbol{C}} \otimes \overline{\mathrm{H}}_{\boldsymbol{C}} \rightarrow \mathbb{C}$ such that

$$
S\left(F^{p}(\mathrm{H}), F^{q}(\overline{\mathrm{H}})\right)=S\left(\bar{F}^{p}(\mathrm{H}), \bar{F}^{q}(\overline{\mathrm{H}})\right)=0 \quad \text { for } \quad p+q>w
$$

(1.3.2) $S(\mathrm{C}(\mathrm{H}) u, \bar{v})$ is a positive definite Hermitian form on $\mathrm{H}_{\mathcal{C}}$.

1.4. A mixed Hodge structure $\mathrm{H}$ of weight $w$ is data $\left(\mathrm{H}_{C}, W(\mathrm{H}), F(\mathrm{H})\right.$, $\bar{F}(\mathrm{H})$ ). Here, $\mathbf{H}_{\boldsymbol{C}}$ is a finite-dimensional $\boldsymbol{C}$-vector space, and $W(\mathrm{H}), F(\mathrm{H})$ and $\bar{F}(\mathrm{H})$ are filtrations of $\mathrm{H}_{C}$ such that $\mathrm{Gr}_{k}^{W}(\mathrm{H})$ is a Hodge structure of weight 
$w+k$ together with the induced filtrations of $F(\mathbb{H})$ and $\bar{F}(\mathbb{H})$. If $w=0$, we call it simply a mixed Hodge structure.

1.5. Let $X$ be a complex manifold and $\bar{X}$ its complex conjugate. A variation of Hodge structure $\mathrm{H}$ is data $\left(\mathbb{H}_{C}, F(H), F(H)\right)$. Here $\mathbb{H}_{C}$ is a locally free $\mathbb{C}_{X}$-module with finite rank and $F(H)$ (resp. $\bar{F}(\mathrm{H})$ ) is a filtration of $\mathcal{O}_{X} \otimes \mathbb{H}_{C}$ (resp. $\left.\mathcal{O}_{\bar{X}} \otimes \mathbb{H}_{C}\right)$ by vector subbundles such that for any $x \in X, \mathbb{H}(x)=\left(\mathbb{H}_{C, x}\right.$, $F(H)(x), \bar{F}(\mathrm{H})(x))$ is a Hodge structure of weight $w$ and $v F^{p}(\mathrm{H}) \subset F^{p-1}(\mathrm{H})$ (resp. $v \bar{F}^{p}(\mathbb{H}) \subset \bar{F}^{p-1}(\mathbb{H})$ ) for any vector field $v$ on $X$ (resp. $\left.\bar{X}\right)$. Here $\bar{X}$ is the complex conjugate of $X$.

A polarization $S$ of a variation of Hodge structure $\mathbb{H}$ is a homomorphism $S: \mathbb{H}_{C} \otimes \bar{H}_{C} \rightarrow C$ such that at each point $x \in X, S_{x}$ gives a polarization of $H(x)$. A variation of Hodge structure is called polarizable if it admits a polarization.

1.6. A variation of mixed Hodge structure $\mathbb{H}$ on $X$ consists of data $\left(\mathbb{H}_{\mathbb{C}}, W(\mathbb{H}), F(H), \bar{F}(H)\right) ; \mathbb{H}_{C}$ is a locally free $\mathbb{C}_{X}$-module of finite rank, $W(\mathbb{H})$ is a filtration of $H_{C}$ by locally free $\mathbb{C}_{X}$-modules and $F(H)$ (resp. $\bar{F}(\mathbb{H})$ ) is a filtration of $\mathcal{O}_{X} \otimes \mathrm{H}_{C}\left(\right.$ resp. $\left.\mathcal{O}_{\bar{X}} \otimes \mathrm{H}_{C}\right)$ by vector subbundles, such that $v \mathbb{F}^{p}(\mathbb{H})$ $\subset \mathbb{F}^{p-1}(\mathbb{H})\left(\right.$ resp. $v \bar{F}^{p}(H) \subset \bar{F}^{p-1}(\mathbb{H})$ ) for any vector field $v$ on $X$ (resp. $\bar{X}$ ) and that $\mathrm{Gr}_{k}^{W(\mathrm{H})}$ is a variation of Hodge structure of weight $k$.

A variation of mixed Hodge structure $\mathbb{H}$ is called graded polarizable if $\mathrm{Gr}_{k}^{W(\mathrm{H})}$ is polarizable for any $k$.

1.7. Let $f: X \rightarrow Y$ be a holomorphic map. Then for a variation of mixed Hodge structure $\mathbb{H}$ on $Y$, we can naturally define $f^{*}(\mathbb{H})$ a variation of mixed Hodge structure on $X$. If $\mathbb{H}$ is graded polarizable, then so is $f^{*}(\mathbb{H})$.

1.8. Let $D$ be the unit disc and $D^{*}$ the punctured unit disc; $D=\{z \in \mathbb{C}$; $|z|<1\}, D^{*}=D \mid\{0\}$. Following Steenbrink-Zucker $[\mathrm{S}-\mathbb{Z}]$, we say that a VMHS (variation of mixed Hodge structure) $\mathrm{H}$ on $D^{*}$ is pre-admissible if it satisfies (1.8.1)-(1.8.4):

(1.8.1) $\mathbb{H}$ is graded polarizable.

(1.8.2) The monodromy $M$ of $\mathbb{H}_{\mathbb{C}}$ around the origin is quasi-unipotent.

(1.8.3) The logarithm $N$ of the unipotent part of $M$ admits a weight filtration relative to $W(\mathrm{H})$ (see $\S 3$ ).

(1.8.4) Let $\mathcal{O}_{D}(\mathbb{H}), W_{k}\left(\mathcal{O}_{D}(\mathbb{H})\right)\left(\operatorname{resp} . \mathcal{O}_{\bar{D}}(\mathbb{H})\right.$ and $\left.W_{k}\left(\mathcal{O}_{\bar{D}}(\mathbb{H})\right)\right)$ be the canonical 
extension of $\mathcal{O}_{D^{*}} \otimes \mathrm{H}_{C}$ and $\mathcal{O}_{D^{*}} \otimes W_{k}(\mathrm{H})$ (resp. $\mathcal{O}_{\bar{D}^{*}} \otimes \mathrm{H}_{C}$ and $\mathcal{O}_{\bar{D}^{*}} \otimes$ $W_{k}(\mathbb{H})$ ). Then $F(\mathbb{H})$ (resp. $\bar{F}(\mathbb{H})$ ) extends to a subbundle of $\mathcal{O}_{D}(\mathbb{H})$ (resp. $\mathcal{O}_{\bar{D}}(\mathrm{H})$ ) such that $\operatorname{Gr}_{F(\mathrm{H})}^{p} \operatorname{Gr}_{k}^{W\left(\mathcal{O}_{D}(\mathrm{H})\right)}$ (resp. $\operatorname{Gr}_{F(\mathrm{H})}^{p} \operatorname{Gr}{ }_{k}^{W\left(O_{\bar{D}}(\mathrm{H})\right)}$ ) is locally free.

1.9. Let $X$ be an analytic space, and $X^{*}$ a non-singular Zariski open subset of $X$. Let $\mathbb{H}$ be a VMHS on $X^{*}$. We say that $H$ is admissible if we have

(1.9.1) $\mathrm{H}$ is graded polarizable.

(1.9.2) For any $f: D \rightarrow X$ such that $f\left(D^{*}\right) \subset X^{*}, f_{0}^{*}(H)$ is pre-admissible. Here $f_{0}: D^{*} \rightarrow X^{*}$ is the restriction of $f$.

When $X=D$ and $X^{*}=D^{*}$ and the monodromy is unipotent, any preadmissible VMHS on $D^{*}$ is admissible by the following lemma.

Lemma 1.9.1. Let $f: D \rightarrow D$ be $z \mapsto z^{n}(n \geqq 1)$ and let $\mathbb{H}$ be a VMHS on $D^{*}$ and $\mathrm{H}^{\prime}=f^{*} \mathrm{H}$. Then we have

(1.9.3) Assume that the monodromy of $\mathrm{H}^{\prime}$ is unipotent. Then the condition (1.8.4) for $\mathrm{H}^{\prime}$ implies (1.8.4) for $\mathrm{H}$.

(1.9.4) Assume that the monodromy of $\mathrm{H}$ is unipotent. Then the condition (1.8.4) for $\mathrm{H}$ implies (1.8.4) for $\mathbb{H}^{\prime}$.

Proof. Let $G=\mathbb{Z}_{n}$ be the Galois group of $f: D \rightarrow D$. Then we have

$$
\begin{aligned}
& \left(f_{*} \mathcal{O}\left(W_{k}\left(\mathrm{H}^{\prime}\right)\right)\right)^{G}=\mathcal{O}\left(W_{k}(\mathrm{H})\right) \quad \text { or } \quad\left(f_{*} z^{-(n-1)} \mathcal{O}\left(W_{k}\left(\mathrm{H}^{\prime}\right)\right)\right)^{G}=\mathcal{O}\left(W_{k}(\mathbb{H})\right), \\
& \left(f_{*} F^{p}\left(\mathrm{H}^{\prime}\right)\right)^{G}=F^{p}(\mathrm{H}) \text { or }\left(f_{*}\left(z^{-(n-1)} F^{p}\left(\mathrm{H}^{\prime}\right)\right)\right)^{G}=F^{p}(\mathrm{H})
\end{aligned}
$$

according to the meaning of canonical extension (i.e. left or right). In either case, (1.8.4) for $\mathrm{H}^{\prime}$ implies (1.8.4) for $\mathrm{H}$ because the $G$-invariant of a vector bundle is also a vector bundle. (1.9.4) follows from the fact

$$
\mathcal{O}\left(W_{k}\left(\mathrm{H}^{\prime}\right)\right)=f^{*} \mathcal{O}\left(W_{k}\left(\mathrm{H}^{\prime}\right)\right) \text { and } F^{p}\left(\mathrm{H}^{\prime}\right)=f^{*} F^{p}(\mathrm{H}) . \quad \text { Q. E. D. }
$$

If $H$ is admissible, then $F^{p}(\mathrm{H})\left(\right.$ resp. $\left.\bar{F}^{p}(\mathrm{H})\right)$ extends to a coherent submodule of the canonical extension of $\mathcal{O}_{X^{*}}(\mathrm{H})\left(\operatorname{resp} . \mathcal{O}_{\bar{X}^{*}}(\mathrm{H})\right)$.

1.10. We conjecture the following

Conjecture: Assume that $X$ is equi-dimensional and let $Z$ be $a$ closed analytic subset of $X$ with codimension $\geqq 2$. If $\left.\mathbb{H}\right|_{X^{*} \backslash Z}$ is admissible in $X \backslash Z$, then $\mathrm{H}$ is admissible in $X$. 
Note that the quasi-unipotency (1.8.2) is a consequence of $[\mathbb{K}]$.

Later, we shall prove this conjecture when $X$ is non-singular and $X \backslash X^{*}$ is a normally crossing hypersurface (Theorem 4.5.2). As a corollary of this theorem, we have

Proposition 1.10.1. Let $X^{*}$ be a non-singular Zariski open subset of a complex analytic variety $X$ and $Y$ a closed analytic subset of $X$ such that $X^{*} \backslash Y$ is dense in $X^{*}$. Then, for any VMHS $\mathbb{H}$ on $X^{*}$ if $\left.\mathbb{H}\right|_{X^{*} \backslash Y}$ is admissible in $X$ then $\mathbb{H}$ is admissible in $X$.

Proof. Let us take $f: X^{\prime} \rightarrow X$ satisfying (1.10.1)-(1.10.4).

(1.10.1) $\quad X^{\prime}$ is non-singular.

(1.10.2) $f$ is proper.

(1.10.3) $f^{-1}\left(X^{*}\right) \rightarrow X^{*}$ is an isomorphism.

(1.10.4) $f^{-1}\left(X \backslash X^{*}\right)$ is a normally crossing hypersurface.

Then $\mathbb{H}$ is admissible if and only if $\left.f^{*} \mathbb{H}\right|_{f^{-1}\left(X^{*}\right)}$ is admissible. In fact, any curve $\varphi: D \rightarrow X$ such that $\varphi\left(D^{*}\right) \subset X^{*}$ comes from a curve in $X^{\prime}$. Similarly $\left.\mathbb{H}\right|_{X^{*} \mid Y}$ is admissible if and only if $\left.f^{*} \mathbb{H}\right|_{f^{-1}\left(X^{*} \mid Y\right)}$ is admissible in $X^{\prime}$. Therefore, by replacing $\mathrm{H}$ with $f^{*} \mathrm{H}$, we may assume from the beginning that $X$ is non-singular and $X \backslash X^{*}$ is a normally crossing hypersurface. Set $Z=\overline{Y \cap X^{*}} \cap\left(X \backslash X^{*}\right)$. Then $\left.\mathbb{H}\right|_{X^{\prime} \backslash Z}$ is admissible in $X \backslash Z$. Since $\operatorname{codim} \mathbb{Z} \geqq 2$, we can apply Theorem 4.5.2.

1.11. Remark also the following.

Proposition 1.11.1. Let us assume $X$ non-singular. If $\operatorname{codim}\left(X \backslash X^{*}\right) \geqq 2$, then a graded polarizable VMHS on $X^{*}$ extends to a graded polarizable VMHS on $X$.

This is a consequence of the following lemma and $\mathbb{W}$. Schmid's theorem [S]: a polarizable variation of Hodge structure outside a closed hypersurface extends to a globally defined polarizable VHS, if the associated local system extends.

Lemma 1.11.2. Let $X$ be a complex manifold, $\mathbb{Z}$ a closed analytic subspace of codimension $\geqq 2$. Let $\mathscr{L}$ be a vector bundle of $\mathbb{X}$ and $\mathbb{W}$ a filtration by subbundles of $\mathscr{L}$. Let $\mathscr{F}$ be a coherent $\mathcal{O}_{X \backslash Z}$ submodule of $\left.\mathscr{L}\right|_{X \backslash Z}$ such that $\mathrm{Gr}_{k}^{W} / \mathscr{F}\left(\mathrm{Gr}_{k}^{W}\right)$ is a locally free $\mathcal{O}_{X \backslash Z}$-module. Assume further that, for any $k$, $\mathscr{F}\left(\mathrm{Gr}_{k}^{W}\right)$ extends to a subbundle of $\mathrm{Gr}_{k}^{W}$ defined on $X$. Then $\mathscr{F}$ extends to a 
subbundle $\widetilde{\mathscr{F}}$ of $\mathscr{L}$ defined on $X$ such that $\widetilde{\mathscr{F}} \mathrm{Gr}_{k}^{W}$ is a subbundle of $\mathrm{Gr}_{k}^{W}$.

Proof. Let $j: X \backslash Z \hookrightarrow X$ be the inclusion map. Set $\widetilde{\mathscr{F}}=j_{*} \mathscr{F} \subset j_{*} j^{-1} \mathscr{L}=\mathscr{L}$. We shall show that $\widetilde{\mathscr{F}} \cap W_{k}$ is a subbundle of $W_{k}$ by the induction on $k$. Consider the following exact sequence

$$
0 \longrightarrow \frac{W_{k-1}}{\widetilde{\mathscr{F}} \cap W_{k-1}} \longrightarrow \frac{W_{k}}{\widetilde{\mathscr{F}} \cap W_{k}} \longrightarrow \frac{G r_{k}^{W}}{\widetilde{\mathscr{F}}\left(G r_{k}^{W}\right)} \longrightarrow 0
$$

By the hypothesis of the induction, $W_{k-1} / \widetilde{\mathscr{F}} \cap W_{k-1}$ is locally free and hence $\mathscr{H}_{Z}^{1}\left(W_{k-1} / \widetilde{\mathscr{F}} \cap W_{k-1}\right)=0$. By the construction of $\widetilde{\mathscr{F}}$ we have $\mathscr{H}_{Z}^{0}\left(W_{k} / \widetilde{\mathscr{F}} \cap W_{k}\right)=0$. Therefore we have $\mathscr{H}_{Z}^{0}\left(\mathrm{Gr}_{k}^{W} / \widetilde{\mathscr{F}}\left(\mathrm{Gr}_{k}^{W}\right)\right)=0$. This means that $\widetilde{\mathscr{F}}\left(\mathrm{Gr}_{k}^{W}\right)$ is the extension as a subbundle of $\mathrm{Gr}_{k}^{W}$. Therefore $\mathrm{Gr}_{k}^{W} / \widetilde{\mathscr{F}} \mathrm{Gr}_{k}^{W}$ is locally free and hence so is $W_{k} / \widetilde{\mathscr{F}} \cap W_{k}$.

Q.E.D.

Similarly, the preceding lemma, together with Schmid's nilpotent orbit theorem [S], implies the following.

Proposition $\mathbb{1}$.11.3. Let $X$ be a complex manifold, $X^{*}$ the complement of a normally crossing hypersurface, $Z$ a closed analytic subset of $X$ of codimension $\geqq 2$ and $\mathrm{H}$ a graded polarizable VMHS on $X^{*}$. Then if $\left.\mathrm{H}\right|_{X^{*} \backslash Z}$ is admissible on $X \backslash Z$, then the Hodge filtration $F(\mathrm{H})$ extends to coherent subsheave of the canonical extension $\mathcal{O}_{X}(\mathrm{H})$ of $\mathcal{O}_{X^{*}} \otimes \mathrm{H}_{C}$ such that $\mathrm{Gr}_{F(\mathrm{H})} \mathrm{Gr}^{W\left(\mathcal{O}_{X}(\mathrm{H})\right)}$ is a locally free $\mathcal{O}_{X}$-module.

\section{§2. Perverse Sheaves in a Normally Crossing Case}

2.1. Let us consider $X=C^{l}$ and for $J \subset I=\{1, \ldots, l\}$, we set $X_{J}=\{z \in X$; $z_{j}=0$ for $\left.j \in J\right\}$ and $X_{J}^{*}=\left\{z \in X_{J} ; z_{j} \neq 0\right.$ for $\left.j \notin J\right\}$. Let $\mathscr{P}$ be the category of perverse sheaves $\mathscr{F} \cdot$ on $X$ such that $\mathscr{F} \cdot \mid X_{J}^{*}$ has locally constant cohomology groups. A. Galligo, M. Granger and $\mathrm{Ph}$. Maisonobe $[\mathrm{G}-\mathrm{G}-\mathrm{M}]$ shows that $\mathscr{P}$ is equivalent to the category $\left\{M_{\alpha} ; f_{\alpha \beta}, g_{\beta \alpha}\right\}$. Here $M_{\alpha}(\alpha \subset I)$ is a vector space and $f_{\alpha \beta}: M_{\beta} \rightarrow M_{\alpha}, g_{\beta \alpha}: M_{\alpha} \rightarrow M_{\beta}$ are given for $\beta \subset \alpha \subset \mathbb{I}$, such that they satisfy

$$
\begin{gathered}
f_{\alpha \beta} \circ f_{\beta \gamma}=f_{\alpha \gamma,} \quad g_{\gamma \beta} g_{\beta \alpha}=g_{\gamma \alpha} \quad \text { for } \alpha \supset \beta \supset \gamma, \\
f_{\alpha \alpha}=g_{\alpha \alpha}=i d, \\
g_{\alpha, \alpha \cup \beta} f_{\alpha \cup \beta, \beta}=f_{\alpha, \alpha \cap \beta} g_{\alpha \cap \beta, \beta} \quad \text { for any } \alpha, \beta, \\
\text { If } \alpha \supset \beta \text { and } \# \alpha=\# \beta+1 \text { then } 1-g_{\beta \alpha} f_{\alpha \beta} \text { is invertible. }
\end{gathered}
$$

Now consider the condition that $\mathscr{F}^{\cdot}$ is the minimal extension of a locally 
constant sheaf on $X_{\gamma}^{*}$. In terms of $\left\{M_{\alpha}\right\}$ this is to saying that $M_{\alpha}=0$ for $\gamma \not \subset \alpha$, and $f_{\alpha \gamma}$ is surjective and $g_{\gamma \alpha}$ is injective for $\gamma \subset \alpha$. If we consider the condition that $\mathscr{F} \cdot$ is a direct sum of such minimal extensions, we arrive to the arguments that we are going to describe in this section.

2.2. Let $f: A \rightarrow B$ and $g: B \rightarrow A$ be morphisms in an abelian category. We write $A \underset{g}{\stackrel{f}{\rightleftarrows}} B$ if we have the following equivalent conditions:

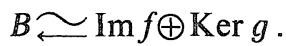$$
\operatorname{Im}(g \circ f) \simeq \operatorname{Im} g \text { and } \operatorname{Ker} f \simeq \operatorname{Ker}(g \circ f) \text {. }
$$

This is a self-dual notion, i.e., if $T$ is an exact contravarient functor, $\underset{\underset{g}{\stackrel{f}{\rightleftarrows}}}{\stackrel{f}{\square}}$ implies $T(A) \underset{T(f)}{\stackrel{T(g)}{\rightleftarrows}} T(B)$

2.3. More generally, let $I$ be a finite set and let $S$ be the set of subsets of $I$. For an abelian category $\mathscr{A}$ let $\mathbb{P}(I, \mathscr{A})$ be the category defined as follows. $\mathrm{Ob}(\mathrm{P}(I, \mathscr{A}))$ consists of $\left\{M_{\alpha}\right\}_{\alpha \in \subseteq}$ and $f_{\alpha \beta}: M_{\beta} \rightarrow M_{\alpha}$ and $g_{\beta \alpha}: M_{\alpha} \rightarrow M_{\beta}$ for $\beta \subset \alpha$ satisfying (2.1.1), (2.1.2) and (2.1.3). The morphisms are defined in an obvious way. For $\gamma \in S$ let $M_{\gamma}(I, \mathscr{A})$ be the set of objects $\left(\left(M_{\alpha}\right),\left(f_{\alpha \beta}\right),\left(g_{\beta \alpha}\right)\right)$ in $\mathbb{P}(I, \mathscr{A})$ satisfying

$$
M_{\alpha}=0 \text { if } \alpha \not \supset \gamma .
$$

$$
f_{\alpha \gamma} \text { is surjective and } g_{\gamma \alpha} \text { is injective if } \alpha \supset \gamma \text {. }
$$

Let $\mathrm{M}(I, \mathscr{A})$ be the set of objects which is isomorphic to the direct sum of objects in $\cup_{\alpha} \mathbb{M}_{\alpha}(I, \mathscr{A})$.

Propositiom 2.3.1. Let $M=\left(\left(M_{\alpha}\right),\left(f_{\alpha \beta}\right),\left(g_{\beta \alpha}\right)\right)$ be an object of $\mathbb{P}(\mathbb{I}, \mathscr{A})$. Then the following conditions are equivalent.

(2.3.3) $M \in M(I, \mathscr{A})$.

(2.3.4) $M_{\beta} \underset{\substack{g_{\beta \alpha}}}{\stackrel{f_{\alpha \beta}}{\rightleftarrows}} M_{\alpha}$ for any $\alpha, \beta$ with $\alpha \supset \beta$.

(2.3.5) (2.3.4) holds for any $\alpha, \beta$ with $\alpha \supset \beta$ and $\# \alpha=\# \beta+1$.

$$
\text { If we set } \mathbb{P}_{\alpha}(M)=\bigcap_{\beta \subsetneq \alpha} \mathbb{K e r} g_{\beta \alpha} \text { then } M_{\alpha} \simeq \underset{\beta \subset \alpha}{\oplus} \int_{\alpha \beta}\left(\mathbb{P}_{\beta}(M)\right) \text { and } g_{\beta \alpha} \text { : }
$$
$f_{\alpha \beta} \mathbb{P}_{\beta}(M) \rightarrow \mathbb{P}_{\beta}(M)$ is injective for $\alpha \supset \beta$. 
Proof. Since $(2.3 .6) \Leftrightarrow(2.3 .3) \Rightarrow(2.3 .4) \Rightarrow(2.3 .5)$ is evident, we shall show (2.3.5) $\Rightarrow(2.3 .3)$. When $|I| \leqq 1$, this is evident. We shall assume $|I|>1$. Taking an element $i_{0}$ of $I$, let $I_{0}=I \backslash\left\{i_{0}\right\}$ and $S_{0}$ the set of subsets of $I_{0}$. Set $\mathscr{B}=$ $\mathbb{P}\left(I_{0}, \mathscr{A}\right)$. Then $\mathbb{P}(I, \mathscr{A})$ is equivalent to $\mathbb{P}\left(\left\{i_{0}\right\}, \mathscr{B}\right)$, by the following functor. For $M=\left(\left(M_{\alpha}\right),\left(f_{\alpha \beta}\right),\left(g_{\beta \alpha}\right)\right) \in \mathrm{Ob}(\mathbb{P}(I, \mathscr{A}))$ set

$$
\begin{aligned}
& i(M)=\left(\left(M_{\alpha}\right),\left(f_{\alpha \beta}\right),\left(g_{\beta \alpha}\right) ; \alpha, \beta \in \mathscr{S}_{0}\right) \in \mathrm{Ob}(\mathscr{B}) \\
& j(M)=\left(M_{\alpha \cup\left\{i_{0}\right\}},\left(f_{\alpha \cup i_{0}, \beta \cup i_{0}}\right),\left(g_{\beta \cup\left\{i_{0}\right\}, \alpha \cup\left\{i_{0}\right\}}\right) ; \alpha, \beta \in \mathscr{S}_{0}\right) \in \mathrm{Ob}(\mathscr{B}) .
\end{aligned}
$$

Then $\left(f_{\alpha \cup\left\{i_{0}\right\}, \alpha}\right)_{\alpha}$ and $\left(g_{\alpha, \alpha \cup\left\{i_{0}\right\}}\right)_{\alpha}$ define $f(M): i(M) \rightarrow j(M)$ and $g(M): j(M) \rightarrow i(M)$, respectively. Thus $t(M)=(i(M), j(M), f(M), g(M))$ gives an object of $\mathbb{P}\left(\left\{i_{0}\right\}\right.$, $\mathscr{B})$. Now assume that $M \in \mathrm{Ob}(P(I, \mathscr{A}))$ satisfies (2.3.5).

Since $t(M)$ satisfies (2.3.5), $t(M)$ belongs to $\mathbb{M}\left(\left(i_{0}\right), \mathscr{B}\right)$. Hence $M \cong$ $M_{1} \oplus M_{2}$ such that $i\left(M_{1}\right)=0$ and $i\left(M_{2}\right) \rightarrow j\left(M_{2}\right)$ is surjective and $j\left(M_{2}\right) \rightarrow i\left(M_{2}\right)$ is injective. As (2.3.5) and (2.3.3) are stable by direct summands, we may assume either $i(M)=0$ or $i(M) \rightarrow j(M) \mapsto i(M)$. In the first case $i(M)=0$, the hypothesis of the induction implies $j(M) \in \mathbb{M}\left(I_{0}, \mathscr{A}\right)$. Hence $M$ belongs to $\mathbb{M}(I, \mathscr{A})$. Therefore, we may assume $i(M) \rightarrow j(M) \mapsto i(M)$, or equivalently, $f_{\alpha \beta}$ is surjective and $g_{\beta \alpha}$ is injective if $\alpha \supset \beta$ and $\alpha \ni i_{0}, \beta \nexists i_{0}$. Now, we may assume this for all $i_{0} \in I$. Hence $M$ belongs to $\mathbb{M}(I, \mathscr{A})$.

Q.E.D.

\section{§ 3. Relative Weight Filltration (cf. $[\mathbb{S}-\mathbb{Z}]$ )}

3.1. Let $W$ be a filtration of an object $\mathbb{H}$ in an abelian category and let $N$ be a nilpotent endomorphism of $\mathrm{H}$ such that $N W_{k} \subset W_{k}$. A relative weight filtration $M$ is a filtration of $\mathbb{H}$ such that

(3.1.1) $N M_{k} \subset M_{k-2}$

(3.1.2) $\quad N^{l}: \mathrm{Gr}_{k+l}^{M} \mathrm{Gr}_{k}^{W} \longrightarrow \mathrm{Gr}_{k-l}^{M} \mathrm{Gr}_{k}^{W}$ is an isomorphism for any $l \geqq 1$ and $k$.

Remark that it is unique if it exists. We shall denote it by $M(N, W)$. As shown in $[\mathbb{S}-\mathbb{Z}]$ we have

(3.1.3) $\quad M_{l}(N, W) \cap W_{k}=W_{k} \cap\left(N^{j}\right)^{-1} M_{l-2 j}(N, W)$ for $j \geqq 0$ and $l \geqq k+j-1$.

(3.1.4) $\quad M_{l}(N, W) \cap W_{k}=N^{j}\left(M_{l+2 j}(N, W) \cap W_{k}\right)+M_{l}(N, W) \cap W_{k-1}$

for $j \geqq 0, \quad l \leqq k-j$.

The last one implies

$$
M(N, W)_{l} \subset N^{r} M(N, W)_{l+2 r}+M(N, W)_{l} \cap W_{l+r-1} \quad \text { for } \quad r \geqq 0 .
$$


Note also the following result in $[S-\mathbb{Z}]$.

$\mathbb{L}$ emmanm 3.1.1 $([S-Z])$. Assume that $\mathbb{H}=\mathbb{W}_{k}$ and that the lweight filtration of $N \mid W_{k-1}$ relative to $W \cap W_{k-1}$ exists. Then the relative weight filtration of $N$ exists if and only if we have

$$
N^{j} W_{k} \cap W_{k-1} \subset N^{j} W_{k-1}+M_{k-j-1}\left(N \mid W_{k-1}, W \cap W_{k-1}\right) \quad \text { for any } j \geqq 1 .
$$

3.2. Now assuming the existence of relative weight filtration, we shall deduce their properties. We denote by $M$ the weight filtration of a nilpotent endomorphism $N$ of $\mathbb{H}$ relative to a filtration $W$ of $\mathbb{H}$. After $[\mathbb{Z}]$, we define

$$
\begin{gathered}
\mathbb{K}_{k}=\mathbb{K}_{k}(W)=\bigcap_{j=0}^{\infty}\left(N^{j}\right)^{-1} W_{k-j}, \\
\mathbb{I}_{k}=\mathbb{I}_{k}(W)=\sum_{j=0}^{\infty} N^{j} W_{k+j} .
\end{gathered}
$$

They are the dual notions of each other. We have

$$
\begin{aligned}
& \mathbb{K}_{k}=W_{k} \cap N^{-1} \mathbb{K}_{k-1}, \\
& \mathbb{I}_{k}=W_{k}+N I_{k+1} .
\end{aligned}
$$

Lemmmæ 3。2。1。 $M_{k}=\sum_{j=o}^{\infty} N^{j}\left(W_{k+j} \cap M_{k+2 j}\right)=\bigcap_{j=0}^{\infty}\left(N^{j}\right)^{-1}\left(W_{k-j}+\mathbb{M}_{k-2 j}\right)$.

Proof. By the duality, it is enough to show

$$
M_{k} \cap \mathbb{W}_{k+r} \subset \sum_{j=0}^{r} N^{j}\left(W_{k+j} \cap M_{k+2 j}\right) \quad \text { for } \quad r \geqq 0 .
$$

We shall prove this by the induction of $r$. This is true for $r=0$ and for $r>0$, we have

$$
\begin{aligned}
& M_{k} \cap W_{k+r} \subset N^{r}\left(W_{k+r} \cap \mathbb{M}_{k+2 r}\right)+M_{k} \cap W_{k+j-1} \\
& \subset N^{r}\left(W_{k+r} \cap M_{k+2 r}\right)+\sum_{j=0}^{r-1} N^{j}\left(W_{k+j} \cap M_{k+2 j}\right)
\end{aligned}
$$

by (3.1.4) and the hypothesis of the induction.

Q. E. D.

Corøllary 3.2.2。 $K_{k} \subset M_{k} \subset \mathbb{I}_{k}$.

Lemma 3.2.3. For $p \geqq q, \mathbb{K}\left(\mathbb{W}\left(\mathbb{W}_{p} / \mathbb{W}_{q}\right)\right)$ and $\mathbb{I}\left(\mathbb{W}\left(\mathbb{W}_{p} / \mathbb{W}_{q}\right)\right)$ are the filtration induced from $\mathbb{K}(W)$ and $\mathbb{I}(W)$, respectively. Here $W\left(W_{p} / W_{q}\right)$ is the filtration of $W_{p} / W_{q}$ induced from $W$.

Proof. It is enough to show 


$$
I_{k} \cap W_{p} \subset \sum_{j=0}^{\infty} N^{j}\left(W_{k+j} \cap W_{p}\right) .
$$

This is trivial for $p \leqq k$. When $p \geqq k$, we shall prove

$$
I_{k} \cap W_{p} \subset \sum_{j=0}^{p-k} N^{j}\left(W_{k+j}\right)
$$

by the descending induction on $p$. We have

$$
I_{k} \cap W_{p} \subset I_{k} \cap W_{p+1} \subset \sum_{j=0}^{p-k+1} N^{j}\left(W_{k+j}\right) .
$$

Therefore, we have

$$
I_{k} \cap W_{p} \subset \sum_{j=0}^{p-k} N^{j} W_{k+j}+N^{p-k+1} W_{p+1} \cap W_{p} .
$$

The last term is contained in $N^{p-k+1} W_{p}+M_{k-1} \cap W_{p}$ by Lemma 2.2.1, and the preceding lemma implies $M_{k-1} \cap W_{p} \subset \sum N^{j}\left(W_{k-1+j} \cap W_{p}\right)$. Thus the induction proceeds.

Lemma 3.2.4. $M_{k} \cap W_{k+r}=N^{r}\left(M_{k+2 r} \cap W_{k+r}\right)+K_{k+r-1} \cap M_{k}$ for $r \geqq 0$ and $M_{k} \cap W_{k+r}=N^{r}\left(M_{k+2 r} \cap W_{k+r}\right)+N^{r-1}\left(M_{k+2 r-2} \cap W_{k+r-1}\right)+K_{k+r-2} \cap M_{k}$ for $r \geqq 1$.

Proof. We have, for $r \geqq 1$

$$
M_{k} \cap W_{k+r} \subset N^{r}\left(M_{k+2 r} \cap W_{k+r}\right)+M_{k} \cap W_{k+r-1} .
$$

Therefore, the second assertion follows from the first. We shall show the first one by the induction on $k$. By the hypothesis of the induction, we have

$$
\begin{aligned}
& N\left(M_{k} \cap W_{k+r-1}\right) \subset M_{k-2} \cap W_{k+r-1} \\
& \subset N^{r+1}\left(M_{k+2 r} \cap W_{k+r-1}\right)+K_{k+r-2} \cap M_{k-2} .
\end{aligned}
$$

Therefore, we have

$$
M_{k} \cap W_{k+r-1} \subset N^{r}\left(M_{k+2 r} \cap W_{k+r-1}\right)+N^{-1} K_{k+r-2} \cap W_{k+r-1} \cap M_{k} .
$$

Since the last term is $K_{k+r-1} \cap M_{k}$ by (3.2.2), we are done.

Q.E.D.

Proposition 3.2.5. $M_{k}=\sum_{j=0}^{\infty} N^{j} K_{k+2 j}=\bigcap_{j=0}^{\infty}\left(N^{j}\right)^{-1} I_{k-2 j}$.

Proof. By the duality, it is enough to show $M_{k} \subset \sum_{j=0}^{\infty} N^{j} K_{k+2 j}$. We shall prove this by the descending induction on $k$. By the preceding lemma, we have

$$
M_{k} \cap W_{k+1} \subset N M_{k+2}+K_{k} .
$$


By (3.1.5) we have

$$
M_{k} \subset N M_{k+2}+M_{k} \cap W_{k} .
$$

Thus, we have

$$
M_{k} \subset N M_{k+2}+K_{k} \subset \sum_{j=1}^{\infty} N^{j} K_{k+2 j}+K_{k} \quad \text { Q.E.D. }
$$

Lemma 3.2.5.1. For $j \geqq 0, W_{k+1} \cap\left(N^{j}\right)^{-1} K_{k} \subset \mathbb{K}_{k+j}$.

Proof. For $0 \leqq v<j$, we have

$$
N^{v}\left(W_{k+1} \cap\left(N^{j}\right)^{-1} K_{k}\right) \subset W_{k+1} \subset W_{k+j-v} .
$$

For $v \geqq j$, we have

$$
N^{v}\left(W_{k+1} \cap\left(N^{j}\right)^{-1} K_{k}\right) \subset N^{v-j} K_{k} \subset W_{k+j-v} .
$$
for $r \geqq 0$.

Lemma 3.2.6. $M_{k} \cap W_{k-r}=\sum_{j=0}^{\infty} N^{j} W_{k-r} \cap N^{-(j+r+1)} K_{k-r-2}+M_{k} \cap W_{k-r-1}$

Proof. We have, by the preceding lemma and Corollary 3.2.2,

$$
N^{j} W_{k-r} \cap N^{-(j+r+1)} K_{k-r-2} \subset N^{j}\left(W_{k-r} \cap N^{-(2 j+r+1)} K_{k-r-1}\right) \subset N^{j} K_{k+2 j} \subset M_{k} .
$$

Therefore, it is enough to show

$$
M_{k} \cap W_{k-r} \subset \sum_{j=0}^{\infty} N^{j} W_{k-r} \cap N^{-(j+r+1)} K_{k-r-2}+W_{k-r-1} .
$$

We shall show this by the descending induction on $r$. Note that (3.2.8) is true if $r$ is so big that $N^{r+1}=0$. Now we have, for any $r \geqq 0$,

$$
\begin{aligned}
N^{1+r}\left(M_{k} \cap W_{k-r}\right) & \subset M_{k-2 r-2} \cap W_{k-r} \\
& \subset N^{r+2}\left(M_{k+2} \cap W_{k-r}\right)+N^{r+1}\left(M_{k} \cap W_{k-r-1}\right)+K_{k-r-2} .
\end{aligned}
$$

by Lemma 3.2.4. This implies

$$
M_{k} \cap W_{k-r} \subset N\left(M_{k+2} \cap W_{k-r}\right)+N^{-(r+1)} K_{k-r-2} \cap W_{k-r}+M_{k} \cap W_{k-r-1} .
$$

The first term of the right hand side is contained, by the hypothesis of the induction, in $N \sum_{j=0}^{\infty} N^{j} W_{k-r} \cap N^{-(r+3+j)} K_{k-r-2} \subset \sum_{j=0}^{\infty} N^{j+1} W_{k-r} \cap N^{-(r+1+j+1)}$. $K_{k-r-2}$ modulo $W_{k-r-1}$. Thus the induction proceeds. $\quad \mathbb{Q} \cdot \mathbb{E} . \mathbb{D}$.

Lemma 3.2.7. For any $r$ and $k$ we have

$$
M_{k} \cap W_{k+r}=\sum_{j, j+r \geqq 0} N^{j+r} W_{k+r} \cap N^{-(j+1)} K_{k+r-2}+M_{k} \cap W_{k+r-1}
$$


Proof. For $r \leqq 0$, this is nothing but the preceding lemma. For $r>0$, we have

$$
\begin{aligned}
M_{k} & \cap W_{k+r}=N^{r}\left(M_{k+2 r} \cap W_{k+r}\right)+M_{k} \cap W_{k+r-1} \\
& =N^{r}\left(\sum_{j=0}^{\infty} N^{j} W_{k+r} \cap N^{-(j+r+1)} K_{k+r-2}+M_{k+2 r} \cap W_{k+r-1}\right)+M_{k} \cap W_{k+r-1} \\
& =\sum_{j=0}^{\infty} N^{j+r} W_{k+r} \cap N^{-(j+1)} K_{k+r-2}+M_{k} \cap W_{k+r-1} \cdot \quad \text { Q. E. }
\end{aligned}
$$

Lemma 3.2.8. Let $\varphi$ be the projection $M_{k} \rightarrow \mathrm{Gr}_{k}^{M}$. Then we have the direct sum decomposition

$$
\varphi\left(M_{k} \cap W_{k+r}\right)=\underset{j, j+r \geqq 0}{\oplus} \varphi\left(N^{j+r} W_{k+r} \cap N^{-(j+1)} K_{k+r-2}\right) \oplus \varphi\left(M_{k} \cap W_{k+r-1}\right) .
$$

Proof. By the preceding lemma it is enough to show that for $m+r, m \geqq 0$

$$
\begin{aligned}
& \quad .9)=N^{m+r} W_{k+r} \cap N^{-(m+1)} K_{k+r-2} \\
& \cap\left(\sum_{0 \leqq j<m, j+r \geqq 0} N^{j+r} W_{k+r} \cap N^{-(j+1)} K_{k+r-2}+M_{k} \cap W_{k+r-1}+M_{k-1}\right) \subset M_{k-1} .
\end{aligned}
$$

In order to see this, it is enough to show

$$
N^{-(m+r)} X \cap W_{k+r} \subset M_{k+2 m+2 r-1} .
$$

By Lemma 3.2.1, this follows from

$$
N^{v}\left(N^{-(m+r)} X \cap W_{k+r}\right) \subset W_{k+2 m+2 r-1-v}+M_{k+2 m+2 r-1-2 v},
$$

which we are going to prove.

For $v \geqq 2 m+r-1$, this follows from

$$
W_{k+r} \subset W_{k+2 m+2 r-1-v} .
$$

If $v \geqq 2 m+r+1>m+r$, we have

$$
\begin{aligned}
N^{v-m-r} X \subset N^{v-m-r-m-1} K_{k+r-2} & \\
& \subset K_{k+r-2-(v-2 m-r-1)}=K_{k+2 m+2 r-v-1} \subset W_{k+2 m+2 r-1-v} .
\end{aligned}
$$

The remaining case is $v=2 m+r$. Then, we have

$$
\begin{aligned}
& N^{v-m-r} X \subset N^{m}\left(\sum_{0 \leqq j<m} N^{-(j+1)} K_{k+r-2}+W_{k+r-1}+M_{k-1}\right) \\
& \quad \subset K_{k+r-2}+W_{k+r-1}+M_{k-2 m-1} \subset W_{k+r-1}+M_{k-2 m-1} \\
& =W_{k+2 m+2 r-1-v}+M_{k+2 m+2 r-1-2 v} .
\end{aligned}
$$

Theorem 3.2.9. We have a canonical decomposition

$$
\mathrm{Gr}_{l}^{M}=\underset{k}{\oplus} \mathrm{Gr}_{k}^{W} \mathrm{Gr}_{l}^{M}
$$


This theorem is one of the key points of this article. (See Remark 5.3.2.)

3.3. Let $W$ and $W^{\prime}$ be a filtration of objects $\mathbb{H}$ and $\mathbb{H}^{\prime}$ in an abelian category, respectively. Let $f: \mathbb{H} \rightarrow \mathbb{H}^{\prime}$ and $g: \mathbb{H}^{\prime} \rightarrow \mathbb{H}$ be morphisms preserving filtrations.

Propositiom 3.3.1. Assume (3.3.1)-(3.3.2):

(3.3.1) $f \circ g$ is nilpotent.

(3.3.2) The weight filtration of $f \circ g$ relative to $\mathbb{W}^{\prime}$ exists.

(3.3.3) For any $k$ we have $\mathrm{Gr}_{k}^{W} \overleftrightarrow{\triangleright} \mathrm{Gr}_{k}^{W^{\prime}}$.

Then the weight filtration of $g \circ f$ relative to $W$ exists and we have

$$
\begin{gathered}
f M_{k}(g \circ f, W) \subset M_{k-1}\left(f \circ g, W^{\prime}\right) . \\
g M_{k}\left(f \circ g, W^{\prime}\right) \subset M_{k-1}(g \circ f, W) .
\end{gathered}
$$

We shall denote $f \circ g$ and $g \circ f$ by the same letter $N$. We shall first show

Lemma 3.3.2. If $M=M(N, W)$ and $M^{\prime}=M\left(N, W^{\prime}\right)$ exist, then we have (3.3.4) and (3.3.5).

Proof. By the duality, it is enough to show one of them, say (3.3.4). We set

$$
K_{k}=\bigcap_{j=0}^{\infty}\left(N^{j}\right)^{-1} W_{k-j} \text { and } K_{k}^{\prime}=\bigcap_{j=0}^{\infty}\left(N^{j}\right)^{-1} \mathbb{W}_{k-j}^{\prime} .
$$

By Proposition 3.2.5, it is enough to show

$$
f \mathbb{K}_{k} \subset \mathbb{K}_{k-1}^{\prime}
$$

We have, for $j \geqq 0$

$$
\left(N^{j} K_{k}\right) \subset W_{k-j} \quad \text { and } \quad(g \circ f)\left(N^{j} K_{k}\right) \subset W_{k-j-j}
$$

Hence (3.3.3) implies $f\left(N^{j} K_{k}\right) \subset W_{k-j-1}^{\prime}$. This shows (3.3.6). Q. E. D.

Now, we shall show the existence of the weight filtration of $N$ relative to $W$. We may assume, by the induction, that $\mathbb{H}=W_{0}, \mathbb{H}^{\prime}=W_{0}^{\prime}$ and that $\tilde{M}=M(N)_{W_{-1}}$, $\left.\left.W\right|_{W_{-1}}\right)$ exists. Therefore, the preceding lemma implies $f \tilde{M}_{k} \subset M_{k-1}^{\prime}$ and $g\left(M_{k}^{\prime} \cap\right.$ $\left.W_{-1}^{\prime}\right) \subset \tilde{M}_{k-1}$. Here $M^{\prime}$ denotes $M\left(N, W^{\prime}\right)$. By Lemma 3.1.1, it is enough to show

$$
\operatorname{Im} N^{j} \cap W_{-1} \subset N^{j} W_{-1}+\tilde{M}_{-j-1} \quad \text { for } \quad j \geqq 1 .
$$

We have, by (3.3.3), 


$$
\begin{aligned}
\operatorname{Im} N^{j} \cap W_{-1} \subset g( & \left.N^{j-1} W_{0}^{\prime} \cap W_{-1}^{\prime}\right) \\
& \subset g\left(N^{j-1} W_{-1}^{\prime}+M_{-j}^{\prime} \cap W_{-1}^{\prime}\right) \subset g N^{j-1} W_{-1}^{\prime}+\tilde{M}_{-j-1} .
\end{aligned}
$$

On the other hand, we have

$$
\begin{aligned}
& W_{k}^{\prime} \subset f W_{k}+W_{k}^{\prime} \cap g^{-1} W_{k-1} \subset f W_{k}+\left(M_{k}^{\prime} \cap W_{k}^{\prime}+W_{k-1}^{\prime}\right) \cap g^{-1} W_{k-1} \\
& \subset f W_{k}+M_{k}^{\prime} \cap W_{k}^{\prime}+W_{k-1}^{\prime} .
\end{aligned}
$$

The induction on $k$ deduced from this

$$
W_{k}^{\prime}=f W_{k}+M_{k}^{\prime} \cap W_{k}^{\prime} .
$$

Therefore, we obtain

$$
\begin{aligned}
& g N^{j-1} W_{-1}^{\prime} \subset g N^{j-1}\left(f W_{-1}+M_{-1}^{\prime} \cap W_{-1}^{\prime}\right) \\
& \subset N^{j} W_{-1}+N^{j-1} \tilde{M}_{-2} \subset N^{j} W_{-1}+\tilde{M}_{-2 j} \subset N^{j} W_{-1}+\tilde{M}_{-j-1} .
\end{aligned}
$$

This shows (3.3.7) and we have completed the proof of Proposition 3.3.1.

Remark that Proposition 3.3.1 means that the existence of relative weight filtration is a micro-local property.

3.4. For a filtration $W$ and a nilpotent endomorphism $N$ such that $N W_{k}$ $\subset W_{k}$, if the relative weight filtration $M(N, W)$ exists, we define the filtration $N_{*} W$ by

$$
\begin{aligned}
\left(N_{*} W\right)_{k} & =N W_{k+1}+M_{k}(N, W) \cap W_{k} \\
& =N W_{k+1}+M_{k}(N, W) \cap W_{k+1} \\
& =N W_{k+1}+\overbrace{j \geqq 0}\left(N^{j}\right)^{-1} W_{k-j} .
\end{aligned}
$$

The first equality follows from (3.1.4) and the second follows from Proposition 3.2.5.

Note that its dual notion is

$$
\begin{aligned}
\left(N_{1} W\right)_{k} & =W_{k-1}+M_{k}(N, W) \cap N^{-1} W_{k-1} \\
& =W_{k-1}+M_{k}(N, W) \cap N^{-1} W_{k-2} .
\end{aligned}
$$

In the sequel, we only treat $N_{*} W$, as we can treat $N_{1} W$ similarly.

Lemma 3.4.1. We have

$$
\left(N W_{k+1}+M(N, W)_{k} \cap W_{k}\right) \cap W_{l}=N\left(W_{k+1} \cap W_{l}\right)+M(N, W)_{k} \cap W_{k} \cap W_{l}
$$

and

$$
\begin{aligned}
& N\left(W_{k+1}+W_{l}\right)+\left(M(N, W)_{k}+W_{l}\right) \cap\left(W_{k}+W_{l}\right) \\
& \quad=N W_{k+1}+M(N, W)_{k} \cap W_{k}+W_{l} .
\end{aligned}
$$


Proof. (3.4.3) is trivial when $l \geqq k+1$. When $l \leqq k$, we shall prove (3.4.3) by the induction on $l$. Writing $M$ for $M(N, W)$, we have

$$
\begin{aligned}
W_{l} & \cap\left(N_{*} W\right)_{k} \subset W_{l+1} \cap\left(N_{*} W\right)_{k} \cap W_{l} \\
\quad & \subset\left(N W_{l+1}+M_{k}\right) \cap W_{l} \subset N\left(M_{k+2} \cap W_{l+1}+W_{l}\right)+M_{k} \subset N W_{l}+M_{k} .
\end{aligned}
$$

Hence we obtain $W_{l} \cap\left(N_{*} W\right)_{k} \subset N W_{l}+M_{k} \cap W_{l}$. The equality (3.4.4) follows from $\left(M(N, W)_{k}+W_{l}\right) \cap\left(W_{k}+W_{l}\right)=M(N, W)_{k} \cap W_{k}+W_{l}$.

Lemma 3.4.2. We have

(0) $N W_{k+1} \cap W_{k} \subset\left(N_{*} W\right)_{k-1}$.

(i) $\mathrm{Gr}_{k+1}^{W} \underset{\triangleright}{\rightleftarrows} \mathrm{Gr}_{k}^{N * W}$.

(ii) The relative weight filtration of $N_{*} W$ is $M(N, W)$.

(iii) $\mathrm{Gr}_{k}^{W} \mathrm{Gr}_{l}^{N * W}= \begin{cases}0 & \text { for } l<k-1 \\ & \text { for } l=k-1\end{cases}$ $N \mathrm{Gr}_{k}^{W}$ for $l=k-1$ $\operatorname{Coker}\left(N ; \operatorname{Gr}_{l+2}^{M(N, W)} \mathrm{Gr}_{k}^{W} \rightarrow \mathrm{Gr}_{l}^{M(N, W)} \mathrm{Gr}_{k}^{W}\right) \quad$ for $\quad l \geqq k$.

Proof. (0) follows from Lemma 3.1.1.

We write $M$ for $M(N, W)$. Then we have

$$
\left(N_{*} W\right)_{k} \cap W_{k}=N W_{k}+M_{k} \cap W_{k} \subset M_{k} \cap W_{k}+\left(N_{*} W\right)_{k-1} .
$$

This shows $\operatorname{Ker}\left(\mathrm{Gr}_{k}^{N_{*} W} \rightarrow \mathrm{Gr}_{k+1}^{W}\right)=M_{k} \cap W_{k} / M_{k} \cap W_{k} \cap\left(N_{*} W\right)_{k-1}$. Now we have

$$
N W_{k+1} \cap\left(M_{k} \cap W_{k}+\left(N_{*} W\right)_{k-1}\right) \subset\left(N_{*} W\right)_{k-1},
$$

In fact, the left hand side of (3.4.6) is contained in $N W_{k+1} \cap W_{k}$.

The property (i) then follows from (3.4.6).

Now we shall prove (ii). It is enough to show the following properties.

$$
M_{k-l} \cap\left(N_{*} W\right)_{k} \subset N^{l}\left(M_{k+l} \cap\left(N_{*} W\right)_{k}\right)+\left(N_{*} W\right)_{k-1} \quad \text { for } l \geqq 1,
$$
and

$$
\left(N_{*} W\right)_{k} \cap\left(N^{l}\right)^{-1}\left(M_{k-l-1}+\left(N_{*} W\right)_{k-1}\right) \subset M_{k+l-1}+\left(N_{*} W\right)_{k-1} \text { for } l \geqq 1 .
$$

We have, by (3.1.4)

$$
\begin{gathered}
M_{k-l} \cap\left(N_{*} W\right)_{k} \subset M_{k-l} \cap W_{k+1} \subset N^{l+1}\left(M_{k+l+2} \cap W_{k+1}\right)+W_{k} \cap M_{k-l} \\
\subset N^{l}\left(M_{k+l} \cap\left(N_{*} W\right)_{k}\right)+\left(N_{*} W\right)_{k-1} .
\end{gathered}
$$

We have also

$$
\begin{aligned}
\left(N_{*} W\right)_{k} & \cap\left(N^{l}\right)^{-1}\left(M_{k-l-1}+\left(N_{*} W\right)_{k-1}\right) \\
& \subset N\left(W_{k+1} \cap\left(N^{l+1}\right)^{-1}\left(M_{k-l-1}+W_{k}\right)\right)+W_{k} \cap M_{k} \\
& \subset N\left(W_{k+1} \cap M_{k+l+1}\right)+N W_{k}+W_{k} \cap M_{k} \subset M_{k+l-1}+\left(N_{*} W\right)_{k-1} .
\end{aligned}
$$


Thus we have (3.4.7) and (3.4.8).

Finally we shall show (iii). By Lemma 3.4.1, we may assume that $W$ is the trivial filtration. Then we have

$$
\left(N_{*} W\right)_{k}=\left\{\begin{array}{ll}
0 & k<-1 \\
\operatorname{Im} N+M_{k}
\end{array} \quad \text { for } \quad k \geqq-1 .\right.
$$

Therefore $\mathrm{Gr}_{-1}^{N * W}=\operatorname{Im} N$ and, for $k \geqq 0$,

$$
\begin{aligned}
\mathrm{Gr}_{k}^{N * W}=\left(\operatorname{Im} N+M_{k}\right) /\left(\operatorname{Im} N+M_{k-1}\right)=M_{k} /\left(\operatorname{Im} N+M_{k-1}\right) \cap M_{k} \\
=M_{k} /\left(M_{k-1}+\operatorname{Im} N \cap M_{k}\right)=M_{k} /\left(M_{k-1}+N M_{k+2}\right) .
\end{aligned}
$$

Thus we obtain (iii).

\section{Corollary 3.4.3。}

$$
\operatorname{Gr}_{k}^{N \star W}=\operatorname{Im}\left(N: \operatorname{Gr}_{k+1}^{W} \rightarrow \operatorname{Gr}_{k+1}^{W}\right) \oplus \operatorname{Coker}\left(N: W_{k} \operatorname{Gr}_{k+2}^{M} \rightarrow W_{k} \mathrm{Gr}_{k}^{M}\right) .
$$

Proof. By the preceding lemma we have

$$
\mathrm{Gr}_{k}^{N * W}=\operatorname{Im}\left(N: \mathrm{Gr}_{k+1}^{W} \rightarrow \mathrm{Gr}_{k+1}^{W}\right) \oplus \operatorname{Ker}\left(\mathrm{Gr}_{k}^{N \star W} \rightarrow \mathrm{Gr}_{k+1}^{W}\right)
$$

The last term is isomorphic to

$$
\left(N_{*} W\right)_{k} \cap W_{k} /\left(N_{*} W\right)_{k-1} \cap W_{k} .
$$

Since $\left(N_{*} W\right)_{k} \cap W_{k} \subset N W_{k+1} \cap W_{k}+W_{k} \cap M_{k} \subset\left(N_{*} W\right)_{k-1}+W_{k} \cap M_{k}$, we have

$$
\begin{aligned}
& \operatorname{Ker}\left(\mathrm{Gr}_{k}^{N * W} \rightarrow \mathrm{Gr}_{k+1}^{W}\right) \cong W_{k} \cap M_{k} / M_{k} \cap\left(N_{*} W\right)_{k-1} \\
& \cong W_{k} \cap M_{k} /\left(M_{k} \cap N W_{k}+M_{k-1} \cap W_{k}\right)=W_{k} \cap M_{k} /\left(N\left(M_{k+2} \cap W_{k}\right)+M_{k-1} \cap W_{k}\right) .
\end{aligned}
$$

This shows the desired result.

\section{§4. Infinitesimal Mixed Hodge Modules}

4.1. Let $w$ be an integer, let $F$ and $\bar{F}$ be two filtrations of a finitedimensional $C$-vector space $\mathrm{H}$, and let $S: \mathrm{H} \otimes \overline{\mathrm{H}} \rightarrow C$ be a non-degenerate bilinear form satisfying

$$
\begin{aligned}
& \overline{S(x, \bar{y}})=(-1)^{w} S(y, \bar{x}) \quad \text { for } \quad x, y \in \mathbb{H} \text {, } \\
& S\left(F^{p},\left(\bar{F}^{q}\right)^{-}\right)=0 \quad \text { for } \quad p+q>w .
\end{aligned}
$$

Let $\left\{N_{1}, \ldots, N_{l}\right\}$ be a mutually commuting set of nilpotent endomorphisms of $\mathrm{H}$ such that $S\left(N_{j} x, \bar{y}\right)+S\left(x, \overline{N_{j} y}\right)=0$ and $N_{j} F^{p} \subset F^{p-1}, N_{j} \bar{F}^{p} \subset \bar{F}^{p-1}$. Then the following conditions are equivalent (essentially due to $[\mathrm{C}-\mathrm{K}]$. See $[\mathbb{K}-\mathbb{K}]$ ). 
(4.1.3) There exists $c$ such that $\left(\mathbb{H}, e^{i \sum t_{J} N_{J} F}, e^{-i \sum t_{J} N_{J}} \bar{F}\right)$ is a Hodge structure of weight $w$ and $S$ polarizes it when $t_{j}>c$.

(4.1.4) The weight filtration $W$ of $N=\sum_{j} t_{j} N_{j}$ does not depend on $t_{1}, \ldots, t_{l}>0$ and $(W, F, \bar{F})$ is a mixed Hodge structure of weight $w$ and the bilinear form $S_{k}: P_{k} \otimes \bar{P}_{k} \rightarrow \mathbb{C}$ given by $S_{k}(x, y)=S\left(x, \overline{N^{k} y}\right)$ polarizes the primitive part $P_{k} \operatorname{Ker}\left(N^{k+1}: \mathrm{Gr}_{k}^{W} \rightarrow \mathrm{Gr}_{-k-2}^{W}\right)$.

If these equivalent conditions are satisfied, we say that $\left(\mathbb{H} ; F, \bar{F} ; N_{1}, \ldots, N_{l} ; S\right)$ is a nilpotent orbit of weight $w$. If $\left(\mathbb{H} ; F, \bar{F} ; N_{1}, \ldots, N_{l} ; S\right)$ is a nilpotent orbit for some $S$, then we say also that $\left(\mathbb{H} ; F, \bar{F} ; N_{1}, \ldots, N_{l}\right)$ is a nilpotent orbit.

4.2. Let $W, F$ and $\bar{F}$ be three filtrations of a finite-dimensional $\mathbb{C}$-vector space $H$ and let $N_{1}, \ldots, N_{l}$ be nilpotent endomorphisms of $\mathbb{H}$ such that

$$
N_{j} F^{p} \subset F^{p-1}, \quad N_{j} \bar{F}^{p} \subset \bar{F}^{p-1} \quad \text { and } \quad N_{j} W_{k} \subset W_{k} .
$$

If $\left(\mathrm{Gr}_{k}^{W}, F\left(\mathrm{Gr}_{k}^{W}\right), \bar{F}\left(\mathrm{Gr}_{k}^{W}\right) ;\left.N_{1}\right|_{\mathrm{Gr}_{k}^{W}}, \ldots,\left.N_{l}\right|_{\mathrm{Gr}_{k}^{W}}\right)$ is a nilpotent orbit of weight $k$ for any $k$, then we say that $\left(\mathbb{H} ; W ; F, \bar{F} ; N_{1}, \ldots, N_{l}\right)$ is a pre-infinitesimal mixed Hodge module (that we shall abbreviate to pre-IMHM, in the sequel).

4.3. Let $\left(\mathbb{H} ; W ; F, \bar{F} ; N_{1}, \ldots, N_{l}\right)$ be a pre-IMHM. Set $I=\{1, \ldots, l\}$. If, for any $J \subset I$, there exists a filtration $M(J)$ of $H$ such that

$$
N_{j} M_{k}(J) \subset M_{k-2}(J) \quad \text { for } j \in J
$$

$$
M(J) \text { is the weight filtration of } \sum_{j \in J} N_{j} \text { relative to } W \text {. }
$$

Then we say that $\left(\mathrm{H} ; W ; F, \bar{F} ; N_{1}, \ldots, N_{l}\right)$ is an infinitesimal mixed Hodge module (abbreviated to IMHM in the sequel). Then by the result of $[\mathbb{C}-\mathbb{K}]$,

(4.3.3) $M(J)$ is the weight filtration of $N$ relative to $W$ for any $N \in \mathbb{C}(J)=$ $\left\{\sum_{j \in J} t_{j} N_{j} ; t_{j}>0\right\}$.

If $\left(\mathbb{H} ; W ; F, \bar{F} ; N_{1}, \ldots, N_{l}\right)$ and $\left(\mathbb{H}^{\prime} ; W^{\prime} ; F^{\prime}, \bar{F}^{\prime} ; N_{1}^{\prime}, \ldots, N_{l}^{\prime}\right)$ are pre-IMHM's, then their tensor product $\left(\mathbb{H} \otimes H^{\prime} ; W \otimes W^{\prime} ; F \otimes F^{\prime}, F \otimes \bar{F}^{\prime} ; N_{1} \otimes N_{1}^{\prime}, \ldots, N_{l} \otimes N_{l}^{\prime}\right)$ and their inner-Hom $\left(\operatorname{Hom}\left(\mathbb{H}, H^{\prime}\right) ; \operatorname{Hom}\left(W, W^{\prime}\right) ; \operatorname{Hom}\left(F, F^{\prime}\right), \operatorname{Hom}\left(\bar{F}, \bar{F}^{\prime}\right)\right.$; $\left.-\operatorname{Hom}\left(N_{1}, N_{1}^{\prime}\right), \ldots,-\operatorname{Hom}\left(N_{l}, N_{l}^{\prime}\right)\right)$ are also pre-IMHM's. The tensor product and the inner-Hom of IMHM's are also IMHM's.

4.4. We shall prove in $\S 6$ the following theorem.

Theorem 4.4 . If. If $\left(\mathbb{H} ; W ; F, \bar{F} ; N_{1}, \ldots, N_{l}\right)$ is a pre-IMHM and if there 
exists the weight filtration of $N_{j}$ relative to $W$ for any $j=1, \ldots, l$, then it is an IMHM.

4.5. Let $X$ be the $n$-dimensional polydisc $D^{n}$ and let $X^{*}$ be $D^{* n}$. Here, $D^{*}$ is the punctured disc $D \backslash\{0\} . \quad$ Let $\mathrm{H}=\left(\mathrm{H}_{C}, W(\mathrm{H}), F(\mathrm{H}), \bar{F}(\mathrm{H})\right)$ be a graded polarizable variation of mixed Hodge structure on $X^{*}$. Letting $\mathcal{O}_{X}(\mathrm{H})$ be a canonical extension of $\mathcal{O}_{X^{*}} \otimes \mathrm{H}_{C}$, we extend $\mathcal{O}_{X^{*}} \otimes W(\mathrm{H})$ as a filtration of subbundles $W\left(\mathcal{O}_{X}(\mathrm{H})\right)$ of $\mathcal{O}_{X}(\mathrm{H})$. Similarly we define $\mathcal{O}_{X}(\mathrm{H})$ and $W\left(\mathcal{O}_{X}(\mathrm{H})\right)$. Let us assume that

(4.5.1) $F(\mathrm{H})\left(\right.$ resp. $\bar{F}(\mathrm{H})$ ) extends to a filtration of $\mathcal{O}_{X}(\mathrm{H})$ (resp. $\mathcal{O}_{\bar{X}}(\mathrm{H})$ ) such

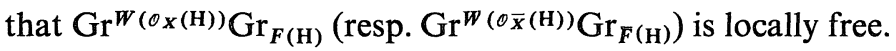

For the sake of simplicity, we assume further

(4.5.2) The monodromy $M_{j}$ of $\mathrm{H}_{C}$ around $z_{j}=0$ is unipotent.

Let $N_{j}$ be the logarithm of $M_{j}$.

Set $U=\left\{z \in X^{*} ; \operatorname{Re} z_{j}>0\right\}$ and we define $\Psi\left(\mathrm{H}_{C}\right)=\Gamma\left(U ; \mathrm{H}_{C}\right)$. We identify $\Psi\left(\mathrm{H}_{C}\right)$ and $C_{\{0\}_{O X}}^{\otimes \mathcal{O}_{X}}(\mathrm{H})$ by $s \mapsto e^{\frac{1}{2 \pi i}} \Sigma N_{j} \log z_{j} s \in \mathcal{O}_{X}(\mathrm{H})$. Then $F(\mathrm{H})$ and $\bar{F}(\mathrm{H})$ give the filtration $F(\Psi(\mathrm{H}))$ and $F\left(\Psi\left(\mathrm{H}_{C}\right)\right)$ of $\Psi\left(\mathrm{H}_{C}\right)$. By the nilpotent orbit theorem ([S]) due to W. Schmid, we have

Proposition 4.5.1. $\Psi(\mathrm{H})=\left\{\Psi\left(\mathrm{H}_{c}\right), \Psi\left(W\left(\mathrm{H}_{C}\right)\right), F(\Psi(\mathrm{H})), \bar{F}(\Psi(\mathrm{H})) ; N_{1}, \ldots\right.$, $\left.N_{n}\right\}$ is a pre-IMHM.

Note that $\mathrm{H}$ is admissible in $X$ if $\Psi(\mathrm{H})$ is an infinitesimal mixed Hodge module. Thus, together with Proposition 1.11.3, Theorem 4.4.1 implies

Theorem 4.5.2. Let $X$ be a complex manifold, $X^{*}$ the complements of a normally crossing hypersurface of $X$ and $Z$ a closed analytic subset of $X$ of codimension $\geqq 2$. If $\mathrm{H}$ is a variation of mixed Hodge structure on $X^{*}$ and if $\left.H\right|_{X^{*} \backslash Z}$ is admissible in $X \backslash Z$, then $\mathrm{H}$ is admissible in $X$.

\section{§5. Properties of Infinitesimal Mixed Hodge Modules}

5.1. In this section, we shall study the properties of infinitesimal mixed Hodge modules. Hereafter we write IMHM for infinitesimal mixed Hodge module. In the course of arguments, we shall make a frequent use of the following 
Theorem 5.1.1. Let $\mathrm{H}=(W(H), F(H), \bar{F}(\mathbb{H}))$ be a mixed Hodge structure. Let $\left\{G_{1}, \ldots, G_{l}\right\}$ be a distributive family (see $[\mathbb{K}]$ ) of filtrations of $\mathbb{H}$ in the category of mixed Hodge structure. Then $\left\{G_{1}, \ldots, G_{l}, \mathbb{W}(\mathbb{H}), \mathbb{F}(\mathbb{H})\right\}$ is a distributive family.

Proof. We shall first show $\left\{G_{1}, \ldots, G_{l}, W(H)\right\}$ is distributive. Since $\left\{G_{1}\right.$ $\left., \ldots, G_{l}\right\}$ is distributive, it is enough to show

$$
\begin{aligned}
& I\left(G_{1}, \ldots, G_{l}\right) \cap W_{k}(H) \\
& =I\left(G_{1} \cap W_{k}(\mathbb{H}), \ldots, G_{l} \cap W_{k}(H)\right) \\
& =S\left(G_{1} \cap W_{k}(\mathrm{H}), \ldots, G_{l} \cap W_{k}(\mathbb{H})\right)
\end{aligned}
$$

(see $[\mathrm{K}]$ ).

This follows from the fact that $\mathbb{H} \mapsto W_{k}(\mathbb{H})$ is an exact functor on the category of mixed Hodge structure. Similarly $\left\{G_{1}, \ldots, G_{l}, \mathbb{F}(H)\right\}$ is a distributive family. Since $W(\mathbb{H})$ is a filtration of $\mathbb{H}$ in the category of mixed Hodge structure, we can argue as $:\left\{G_{1}, \ldots, G_{l}\right\}$ is distributive $\Rightarrow\left\{G_{1}, \ldots, G_{l}, W(\mathbb{H})\right\}$ is distributive $\Rightarrow\left\{G_{1}, \ldots\right.$, $\left.G_{l}, W(\mathbb{H}), F(\mathbb{H})\right\}$ is distributive.

5.2. The following proposition is proved by Deligne (see $[S-\mathbb{Z}]$ ). We shall give another proof.

Proposition 5.2.1. Let $W, F, \bar{F}$ be filtrations of $\mathbb{H}$ and let $N$ be a nilpotent endomorphism of $\mathbb{H}$ satisfying

$$
N F^{p} \subset F^{p-1}, \quad N \bar{F}^{p} \subset \bar{F}^{p-1} \text { and } N W_{k} \subset W_{k} .
$$

Assume further

(5.2.1) There exists the weight filtration $M$ of $N$ relative to $W$.

(5.2.2) $\mathrm{Gr}_{k}^{W}$ is a mixed Hodge structure with the induced filtration of $M$ as a weight filtration and with the induced filtrations from $F$ and $\bar{F}$ as Hodge filtration.

Then, $(M ; F, \bar{F})$ is a mixed Hodge structure.

Proof. By the induction, we may assume that

$$
\begin{aligned}
& H=W_{0} \\
& \left(M \cap W_{k} ; F \cap W_{k}, \bar{F} \cap W_{k}\right)
\end{aligned}
$$

is a mixed Hodge structure if $k<0$.

Lemma 5.2.2. (M, F, W) is a distributive family of filtrations. 
By Theorem 5.1.1, $\left(M \cap W_{-1}, F \cap W_{-1}, W \cap W_{-1}\right)$ is distributive. Hence it is enough to show, for $k \geqq 1$

$$
\begin{aligned}
& M_{k-1} \cap\left(F^{p}+W_{-1}\right) \subset M_{k-1} \cap F^{p}+M_{k-1} \cap W_{-1} . \\
& M_{-k} \cap\left(F^{p}+W_{-1}\right) \subset M_{-k} \cap F^{p}+M_{-k} \cap W_{-1} .
\end{aligned}
$$

We shall show $\left(A_{k+1}\right) \Rightarrow\left(B_{k}\right)$ for $k \geqq 1$ and $\left(A_{k+2}\right) \Rightarrow\left(A_{k}\right)$ for $k \geqq 1$. They establish $\left(A_{k}\right)$ and $\left(B_{k}\right)$ for all $k$.

$$
\begin{aligned}
&\left(A_{k+1}\right) \Rightarrow\left(B_{k}\right): \text { Since }\left(F^{p} \cap M_{-k}\right)\left(\mathrm{Gr}_{0}^{W}\right)=N^{k}\left(F^{p+k} \cap M_{k}\right)\left(\mathrm{Gr}_{0}^{W}\right), \text { we have } \\
& M_{-k} \cap\left(F^{p}+W_{-1}\right) \subset N^{k}\left(M_{k} \cap\left(F^{p+k}+W_{-1}\right)\right)+W_{-1} \\
& \subset N^{k}\left(M_{k} \cap F^{p+k}+M_{k} \cap W_{-1}\right)+W_{-1} \subset M_{-k} \cap F^{p}+W_{-1}
\end{aligned}
$$

$\left(\mathrm{A}_{k+2}\right) \Rightarrow\left(A_{k}\right):$ we have

$$
\begin{aligned}
& N^{k}\left(F^{p} \cap\left(M_{k-1}+W_{-1}\right)\right) \subset F^{p-k} \cap\left(M_{-k-1}+W_{-1}\right) \\
& \subset N^{k+1}\left(F^{p+1} \cap\left(M_{k+1}+W_{-1}\right)\right)+W_{-1} \subset N^{k+1}\left(F^{p+1} \cap M_{k+1}\right)+W_{-1} \\
& \subset N^{k}\left(F^{p} \cap M_{k-1}\right)+W_{-1} .
\end{aligned}
$$

Furthermore we have

$$
N^{k}\left(F^{p} \cap\left(M_{k-1}+W_{-1}\right)\right) \cap W_{-1} \subset F^{p-k} \cap\left(N^{k} W_{-1}+M_{-k-1} \cap W_{-1}\right) .
$$

Since $N^{k} W_{-1}$ is also a mixed Hodge structure, $\left\{N^{k} W_{-1}, F^{p-k} \cap W_{-1}, M_{-k-1} \cap\right.$ $\left.W_{-1}\right\}$ is distributive by Theorem 5.1.1. Thus the right hand-side of (5.2.6) is contained in $F^{p-k} \cap N^{k} W_{-1}+F^{p-k} \cap M_{-k-1} \cap W_{-1}=N^{k}\left(F^{p} \cap W_{-1}\right)+F^{p-k} \cap$ $M_{-k-1} \cap W_{-1}$.

Finally, (5.2.5) shows

$$
\begin{aligned}
F^{p} \cap\left(M_{k-1}+W_{-1}\right) \subset F^{p} \cap M_{k-1}+F^{p} \cap W_{-1} \\
+\left(N^{k}\right)^{-1} M_{-k-1} \subset M_{k-1}+F^{p} \cap W_{-1} .
\end{aligned}
$$

Q.E.D.

Now, we shall prove the proposition 5.2.1. By the assumption $\left(\operatorname{Gr}_{l}^{M} \operatorname{Gr}_{k}^{W}\right.$, $\left.F\left(\mathrm{Gr}_{l}^{M} \mathrm{Gr}_{k}^{W}\right), \bar{F}\left(\mathrm{Gr}_{l}^{M} \mathrm{Gr}_{k}^{W}\right)\right)$ is a Hodge structure of weight $l$ for any $l$. By the preceding proposition, we have $F\left(\mathrm{Gr}_{l}^{M} \mathrm{Gr}_{k}^{W}\right)=F\left(\mathrm{Gr}_{k}^{W} \mathrm{Gr}_{l}^{M}\right)$, which implies $\left(\mathrm{Gr}_{l}^{M}\right.$, $\left.F\left(\mathrm{Gr}_{l}^{M}\right), \bar{F}\left(\mathrm{Gr}_{l}^{M}\right)\right)$ is a Hodge structure of weight $l$ because an extension of Hodge structures of weight $l$ is also a Hodge structure of the same weight. This completes the proof of Proposition 5.2.1.

As a corollary of Proposition 5.2.1, we have

Proposition 5.2.3. If $\left(W ; F, \bar{F} ; N_{1}, \ldots, N_{l}\right)$ is an IMHM, then $(M(\{1, \ldots, l\})$, 
$F, \bar{F})$ is a mixed Hodge structure. Moreover $N_{j}$ is a morphism of mixed Hodge structure (up to the Tate twist).

In particular, $M(J), W$, etc. are filtrations in the category of mixed Hodge structures.

Propositiom 5.2.4. Let $\left(W ; F, \bar{F} ; N_{1}, \ldots, N_{l}\right)$ be an IMHM, and let $J_{1} \subset \cdots \subset J_{k} \subset\{1, \ldots, l\}$ be an increasing sequence. Then $\left\{W, F, M\left(J_{1}\right), \ldots\right.$, $\left.M\left(J_{k}\right)\right\}$ is distributive.

Proof. We shall prove this by the induction of $k$. We may assume that $J_{k}=\{1, \ldots, l\}$ and that $\left\{W, M\left(J_{1}\right), \ldots, M\left(J_{k-1}\right)\right\}$ is distributive. Since $W$ and $M\left(J_{p}\right)(1 \leqq p<k)$ are filtrations of $\left(M\left(J_{k}\right) ; F, \bar{F}\right)$ in the category of mixed Hodge structure, $\left\{W, F, M\left(J_{1}\right), \ldots, M\left(J_{k}\right)\right\}$ is distributive by Theorem 5.1.1.

The following is proved by Cattani-Kaplan $[\mathbb{C}-\mathbb{K}]$ in the pure case and asked by Steenbrink-Zucker $([\mathrm{S}-\mathbb{Z}])$ in the mixed case.

Proposition 5.2.5. Let $\left(W ; F, \bar{F} ; N_{1}, \ldots, N_{l}\right)$ be an IMHM. Then, for $J_{1}, J_{2} \subset\{1, \ldots, l\}$ and $N \in \mathbb{C}\left(J_{2}\right)=\left\{\sum_{j \in J_{2}} t_{j} N_{j} ; t_{j}>0\right\}, M\left(J_{1} \cup J_{2}\right)$ is the weight filtration of $N$ relative to $M\left(J_{1}\right)$.

Proof. We have $N M_{k}\left(J_{1} \cup J_{2}\right) \subset M_{k-2}\left(J_{1} \cup J_{2}\right)$. Hence it is enough to show that

$$
N^{l}: \operatorname{Gr}_{k+l}^{M\left(J_{1} \cup J_{2}\right)} \mathrm{Gr}_{k}^{M\left(J_{1}\right)} \longrightarrow \operatorname{Gr}_{k-l}^{M\left(J_{1} \cup J_{2}\right)} \mathrm{Gr}_{k}^{M\left(J_{1}\right)}
$$

is an isomorphism. This follows from the fact that $\left\{M\left(J_{1} \cup J_{2}\right), M\left(J_{1}\right), W\right\}$ is a distributive family and the fact that

$$
N^{l}: \operatorname{Gr}_{k+l}^{M\left(J_{1} \cup J_{2}\right)} \operatorname{Gr}_{k}^{M\left(J_{1}\right)} \mathrm{Gr}_{p}^{W} \longrightarrow \operatorname{Gr}_{k-l}^{M\left(J_{1} \cup J_{2}\right)} \mathrm{Gr}_{k}^{M\left(J_{1}\right)} \mathrm{Gr}_{p}^{W}
$$

is an isomorphism, which is due to Cattani-Kaplan $[\mathrm{C}-\mathrm{K}]$.

Proposition 5.2.6. (i) The category of pre-IMHM's is an abelian category.

(ii) The category of IMHM's is abelian, on which $W, M\left(N_{1}, W\right), \mathrm{Gr}^{W}$, $\mathrm{Gr}^{M\left(N_{1}, W\right)}$, etc., are exact functors

Proof. (i) is almost evident. We shall show (ii). The problem is only the existence of relative weight filtrations of the kernel, the cokernel, etc. Therefore it is enough to show that for a morphism $f: \mathbb{H}=(W(H), F(H), \bar{F}(\mathbb{H})$; $\left.N_{1}, \ldots, N_{l}\right) \rightarrow \mathbb{H}^{\prime}=\left(W\left(\mathbb{H}^{\prime}\right), F(\mathbb{H}), \bar{F}\left(\mathbb{H}^{\prime}\right) ; N_{1}, \ldots, N_{l}\right)$ in the category of IMHM's, the restriction of $M\left(N_{1}, W(\mathbb{H})\right)$ to $\operatorname{Ker} f$ is the weight filtration of $N_{1}$ relative to $W(\mathbb{H}) \cap \operatorname{Ker} f$. Set $\mathbb{H}^{\prime \prime}=\operatorname{Ker} f$. Now $\mathrm{H}$ and $\mathbb{H}^{\prime}$ have a mixed Hodge structure 
with $W$ as a weight filtration and $e^{i \sum t_{j} N_{j} F}$ and $e^{-i \Sigma t_{j} N_{J}} \bar{F}$ as Hodge filtrations for $t_{1}, \ldots, t_{\iota} \gg 0$, respectively. Hence $f$ is strict with respect to $W$-filtration. On the other hand, $\mathrm{H}$ and $\mathrm{H}^{\prime}$ have another mixed Hodge structure with $M\left(N_{1}, W\right)$ as a weight filtration, respectively. Moreover, $W$ is a filtration of them by submixed Hodge structures. Therefore if we denote by $M\left(\mathbb{H}^{\prime \prime}\right)$ and $W\left(\mathbb{H}^{\prime \prime}\right)$ the restriction of $M\left(N_{1}, W(\mathrm{H})\right)$ and $W(\mathrm{H})$ to $\mathrm{H}^{\prime \prime}$, then the rows in the following diagram are exact.

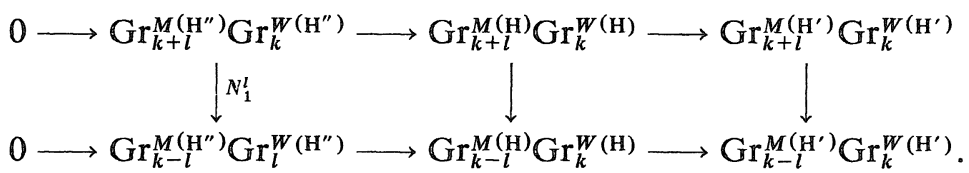

Here $M(\mathrm{H})=M\left(N_{1}, W(\mathrm{H})\right)$ and $M\left(\mathrm{H}^{\prime}\right)=M\left(N_{1}, W\left(\mathrm{H}^{\prime}\right)\right)$. Since the vertical arrows are isomorphisms except the left one, the left one is also an isomorphism. This shows that $M\left(\mathbb{H}^{\prime \prime}\right)$ is the weight filtration of $N_{1}$ relative to $W\left(\mathbb{H}^{\prime \prime}\right)$. Q. E. D.

5.3. As an application of Theorem 3.2.9, we shall prove the following

Proposition 5.3.1. Let $\left(W ; F, \bar{F} ; N_{1}, \ldots, N_{l}\right)$ be a pre-IMHM. Assume that the weight filtration $M$ of $N_{1}$ relative to $W$ exists. Let $N_{1 *} W$ be the filtration as defined in (3.4.1). Then $\left(M ; F, \bar{F} ; N_{2}, \ldots, N_{l}\right)$ and $\left(N_{1 *} W ; F, \bar{F}\right.$; $\left.N_{1}, N_{2}, \ldots, N_{l}\right)$ are pre-IMHM's.

Proof. In order to show the first, we have to prove the existence of a polarization on $\mathrm{Gr}_{l}^{M}$, by which $(F(t), \bar{F}(t))=\left(e^{i \sum t_{j} N_{j}} F, e^{-i \sum t_{j} N_{j}} \bar{F}\right)$ is polarized for $t_{1}, \ldots, t_{l} \gg 0$. Now by Theorem 3.2.9, $\mathrm{Gr}_{l}^{M}$ is a product of $\mathrm{Gr}_{l}^{M} \mathrm{Gr}_{k}^{W}$ 's canonically. This is true also as a Hodge structure with $(F(t), \bar{F}(t))$ as Hodge filtrations. On the other hand, $\mathrm{Gr}_{l}^{M} \mathrm{Gr}_{k}^{W}$ has a polarization free from $t$, by which $F(t)$ is polarized. Therefore $\operatorname{Gr}_{l}^{M}$ has a polarization by which $(F(t), \bar{F}(t))$ is polarized. Thus, $\left(M ; F, \bar{F} ; N_{2}, \ldots, N_{l}\right)$ is a pre-IMHM. Now we shall show $\left(N_{1 *} W ; F, \bar{F} ; N_{1}, N_{2}, \ldots, N_{l}\right)$ is a pre-IMHM. We have by Corollary 3.4 .3

$$
\mathrm{Gr}_{k}^{N_{1 *} W}=N_{1} \operatorname{Gr}_{k+1}^{W} \oplus \operatorname{Coker}\left(N_{1}: W_{k} \mathrm{Gr}_{k+2}^{M} \rightarrow W_{k} \mathrm{Gr}_{k}^{M}\right) \text {. }
$$

Since $\operatorname{Gr}_{k}^{M}$ is polarized, so is $\operatorname{Coker}\left(N_{1}: W_{k} \mathrm{Gr}_{k+2}^{M} \rightarrow W_{k} \mathrm{Gr}_{k}^{M}\right)$. Since $\left(\mathrm{Gr}_{k+1}^{W} ; F\right.$, $\left.\bar{F} ; N_{1}, \ldots, N_{l}\right)$ is a nilpotent orbit, the vanishing cycle theorem ( $[\mathrm{K}-\mathrm{K}],[\mathrm{C}-\mathrm{K}-\mathrm{S}]$ ) shows that $\left(N_{1} \mathrm{Gr}_{k+1}^{W} ; F, \bar{F} ; N_{1}, \ldots, N_{l}\right)$ is also a nilpotent orbit.

Remark 5.3.2. Similarly to Proposition 5.3.1, Theorem 3.2.9 implies the following. Let $X$ be a complex manifold, $f: X \rightarrow C$ be a holomorphic map 
such that $f^{-1}(0)$ is non-singular. Let $\mathbb{H}$ be a graded polarizable VMHS on $X \backslash f^{-1}(0)$ admissible on $X$. Then the near-by cycle of $\mathbb{H}$ with respect to $f$ is also a graded polarizable VMHS.

In the following sections 5.4 and 5.5, we shall give the weight filtration relative to $N_{1 *} W$.

5.4. Let $\left(\mathbb{H} ; F, \bar{F} ; N_{1}, N_{2}\right)$ be a nilpotent orbit of weight $w$. Set $\mathbb{W}(\mathbb{H})$ $=M\left(N_{1}+N_{2}\right), W^{\prime}=M\left(N_{1}\right)$. Here, $M\left(^{*}\right)$ shows the weight filtration.

Lemma 5.4.1. $\quad \operatorname{Ker} N_{1}^{k} \cap \operatorname{Im} N_{2} \subset N_{2}\left(\operatorname{Ker} N_{1}^{k}\right)+W_{k-2}$

$$
\operatorname{Im} N_{1}^{k} \cap \operatorname{Im} N_{2} \cap W_{-k-1} \subset \operatorname{Im} N_{2} N_{1}^{k} \text {. }
$$

Proof. By the purity theorem $([\mathrm{K}-\mathrm{K}],[\mathrm{C}-\mathrm{K}-\mathrm{S}])$ we have

$$
\operatorname{Ker} N_{1}^{k} N_{2} \subset \operatorname{Ker} N_{1}^{k-1} N_{2}+\operatorname{Ker} N_{1}^{k}+W_{k} .
$$

Therefore the induction on $k$ shows

$$
\operatorname{Ker} N_{1}^{k} N_{2} \subset \operatorname{Ker} N_{1}^{k} \operatorname{Ker} N_{2}+W_{k} .
$$

This shows the first statement. The second statement is the dual one.

Proposition 5.402.

$$
\begin{aligned}
M_{k}\left(N_{1} \mid \operatorname{Im} N_{2}\right)=\left(N_{2 *} M\left(N_{1}\right)\right)_{k-1} \cap \operatorname{Im} N_{2} & =N_{2} W_{k}^{\prime}+W_{k-1} \cap W_{k-1}^{\prime} \cap \operatorname{Im} N_{2} \\
& =N_{2} W_{k}^{\prime}+W_{k-1} \cap W_{k}^{\prime} \cap \operatorname{Im} N_{2} .
\end{aligned}
$$

Proof. Let $\widetilde{W}_{k}$ be $\left(N_{2 *} M\left(N_{1}\right)\right)_{k-1} \cap \mathbb{I m} N_{2}$. It is enough to show (5.4.1)(5.4.3).

$$
\begin{aligned}
& N_{1} \widetilde{W}_{k} \subset \widetilde{W}_{k-2} \\
& \widetilde{W}_{-k} \subset N_{1}^{k} \widetilde{W}_{k} \quad \text { for } \quad k \geqq 1 . \\
& \operatorname{Ker} N_{1}^{k} \cap \operatorname{Im} N_{2} \subset \widetilde{W}_{k-1} \quad \text { for } \quad k \geqq 1 .
\end{aligned}
$$

The first one is obvious. The last one follows from the preceding lemma. We shall show the second. In the expression $\tilde{W}_{-k}=N_{2} W_{-k}^{\prime}+\mathbb{W}_{-k-1} \cap W_{-k}^{\prime} \cap$ $\operatorname{Im} N_{2}$, the first term is contained in $N_{2} N_{1}^{k} W_{k}^{\prime} \subset N_{1}^{k} \widetilde{W}_{k}$ and the second term is contained in

$$
\begin{aligned}
W_{-k-1} \cap \operatorname{Im} N_{1}^{k} \cap \operatorname{Im} & N_{2} \cap W_{-k}^{\prime} \subset \operatorname{Im} N_{1}^{k} N_{2} \cap W_{-k-1} \cap W_{-k}^{\prime} \\
& \subset N_{1}^{k} N_{2}\left(W_{k+1}\right) \cap W_{-k}^{\prime} \subset N_{1}^{k}\left(N_{2} W_{k+1} \cap W_{k}^{\prime}\right) \subset N_{1}^{k} \widetilde{W}_{k} .
\end{aligned}
$$

5.5. Let $\left(W ; F, \bar{F} ; N_{1}, N_{2}\right)$ be an $\mathbb{I M H M}$. 


\section{Theorem 5.5.1.}

$$
N_{2 *} M\left(N_{1}, W\right)=M\left(N_{1}, N_{2 *} W\right) .
$$

We shall reduce this theorem to Proposition 5.4.2. In order to perform this smoothly, we shall first show that these filtrations commutes with $\mathrm{Gr}^{W}$.

For simplicity, we write

$$
\begin{aligned}
& M^{\prime}=M\left(N_{1}, W\right), \\
& M^{\prime \prime}=M\left(N_{2}, W\right), \quad M=M\left(N_{1}+N_{2}, W\right)=M\left(N_{2}, M^{\prime}\right) \\
& \tilde{W}^{\prime}=N_{1 *} W, \quad \widetilde{W}^{\prime \prime}=N_{2 *} W \text { and } \tilde{M}=N_{2 *} M^{\prime} .
\end{aligned}
$$

Hence we have

$$
\begin{aligned}
& \tilde{W}_{k}^{\prime \prime}=N_{2} W_{k+1}+M_{k}^{\prime \prime} \cap W_{k}, \\
& \tilde{M}_{k}=N_{2} M_{k+1}^{\prime}+M_{k} \cap M_{k}^{\prime} .
\end{aligned}
$$

Lemma 5.5.2. (i) $\widetilde{W}_{k}^{\prime \prime} \cap W_{a}=N_{2}\left(W_{k+1} \cap W_{a}\right)+M_{k}^{\prime \prime} \cap W_{k} \cap W_{a}$.

(ii) $\tilde{W}_{k}^{\prime \prime}+W_{a}=N_{2}\left(W_{k+1}+W_{a}\right)+\left(M_{k}^{\prime \prime}+W_{a}\right) \cap\left(W_{k}+W_{a}\right)$.

(iii) $\tilde{M}_{l} \cap W_{a}=N_{2}\left(M_{l+1}^{\prime} \cap W_{a}\right)+M_{l} \cap M_{l}^{\prime} \cap W_{a}$.

(iv) $\tilde{M}_{l}+W_{a}=N_{2}\left(M_{l+1}^{\prime}+W_{a}\right)+\left(M_{l}+W_{a}\right) \cap\left(M_{l}^{\prime}+W_{a}\right)$.

Proof. (i) follows from Lemma 3.4.1.

(ii) is trivial.

(iv) follows from the fact that $\left\{M, M^{\prime}, W\right\}$ is distributive (Proposition 5.2.3)

Finally, we shall show (iii). Since $M\left(H / W_{a}\right)=M\left(N_{2}, M^{\prime}\left(H / W_{a}\right)\right)$, we have, using (3.1.3) and the fact that $\left\{W, M, M^{\prime}\right\}$ is distributive,

$$
\begin{aligned}
\tilde{M}_{l} \cap W_{a}= & \left(N_{2} M_{l+1}^{\prime}+M_{l}^{\prime} \cap M_{l}\right) \cap W_{a} \\
& \subset\left(N_{2}\left(M_{l+1}^{\prime} \cap\left(M_{l+2}+W_{a}\right)+M_{l+1}^{\prime} \cap W_{a}\right)+M_{l}^{\prime} \cap M_{l}\right) \cap W_{a} \\
& \subset N_{2}\left(M_{l+1}^{\prime} \cap W_{a}\right)+M_{l} \cap M_{l+1}^{\prime} \cap W_{a} \\
= & N_{2}\left(M_{l+1}^{\prime} \cap W_{a}\right)+M_{l} \cap M_{l}^{\prime} \cap W_{a} .
\end{aligned}
$$

Lemma 5.5.3。 $\left\{\tilde{M}, W, \tilde{W}^{\prime \prime}\right\}$ is distributive.

Proof. It is enough to show

$$
\tilde{M}_{l} \cap\left(W_{a}+\tilde{W}_{k}^{\prime \prime}\right) \subset \tilde{M}_{l} \cap W_{a}+\tilde{M}_{l} \cap \tilde{W}_{k}^{\prime \prime} .
$$

When $a \geqq k+1$, this is trivial because $W_{a} \supset \widetilde{W}_{k}^{\prime \prime}$. So, assume $a \leqq k$. Then we have

$$
\begin{aligned}
\tilde{M}_{l} \cap\left(W_{a}+\tilde{W}_{k}^{\prime \prime}\right) \subset\left(N_{2} M_{l+1}^{\prime}+M_{l}\right) & \cap\left(W_{a}+\tilde{W}_{k}^{\prime \prime}\right) \\
& \subset M_{l} \cap\left(W_{a}+\tilde{W}_{k}^{\prime \prime}\right)+N_{2} M_{l+1}^{\prime} \cap\left(W_{a}+\tilde{W}_{k}^{\prime \prime}\right)
\end{aligned}
$$


because $\left\{M, N_{2} M^{\prime}, W_{a}+\widetilde{W}_{k}^{\prime \prime}\right\}$ is distributive by Theorem 5.1.1. Since $\operatorname{Ker}\left(N_{2} \mid\right.$ $\left.M_{l+1}^{\prime}\left(\mathrm{H} / W_{k+1}\right)\right) \subset M_{l+1}\left(\mathrm{H} / W_{k+1}\right)$, we have

$$
\begin{aligned}
& N_{2} M_{l+1}^{\prime} \cap\left(W_{a}+\tilde{W}_{k}^{\prime \prime}\right) \subset N_{2} M_{l+1}^{\prime} \cap W_{k+1} \cap\left(W_{a}+\widetilde{W}_{k}^{\prime \prime}\right) \\
& \quad \subset N_{2}\left(M_{l+1}^{\prime} \cap M_{l+1}+M_{l+1}^{\prime} \cap W_{k+1}\right) \cap\left(W_{a}+\tilde{W}_{k}^{\prime \prime}\right) \\
& \quad \subset N_{2}\left(M_{l+1}^{\prime} \cap W_{k+1}\right)+M_{l} \cap\left(W_{a}+\tilde{W}_{k}^{\prime \prime}\right) \subset \tilde{M}_{l} \cap \tilde{W}_{k}^{\prime \prime}+M_{l} \cap\left(W_{a}+\widetilde{W}_{k}^{\prime \prime}\right) .
\end{aligned}
$$

Hence we have

$$
\tilde{M}_{l} \cap\left(W_{a}+\tilde{W}_{k}^{\prime \prime}\right) \subset \tilde{M}_{l} \cap \tilde{W}_{k}^{\prime \prime}+M_{l} \cap M_{l+1}^{\prime} \cap\left(W_{a}+\tilde{W}_{k}^{\prime \prime}\right) .
$$

Since $M_{l} \cap W_{a} \subset N_{2}\left(M_{l+2} \cap W_{a}\right)+M_{l} \cap M_{l}^{\prime} \cap W_{a} \subset \tilde{W}_{k}^{\prime \prime}+M_{l} \cap M_{l}^{\prime} \cap W_{a}$, we have

$$
\begin{aligned}
M_{l} \cap M_{l+1}^{\prime} \cap & \left(W_{a}+\tilde{W}_{k}^{\prime \prime}\right) \subset M_{l+1}^{\prime} \cap\left(M_{l} \cap W_{a}+M_{l} \cap \tilde{W}_{k}^{\prime \prime}\right) \\
& \subset M_{l+1}^{\prime} \cap\left(M_{l} \cap M_{l}^{\prime} \cap W_{a}+M_{l} \cap \tilde{W}_{k}^{\prime \prime}\right) \\
& \subset M_{l} \cap M_{l}^{\prime} \cap W_{a}+M_{l} \cap M_{l+1}^{\prime} \cap \tilde{W}_{k}^{\prime \prime} \subset \tilde{M}_{l} \cap W_{a}+\tilde{M}_{l} \cap \tilde{W}_{k}^{\prime \prime} .
\end{aligned}
$$

Q.E.D.

Now, we are ready to prove Theorem 5.5.1. In order to prove this it is enough to show

$$
N_{1}^{l}: \operatorname{Gr}_{k+l}^{\widetilde{T}} \operatorname{Gr}_{k}^{\widetilde{W}} \longrightarrow \operatorname{Gr}_{k-l}^{\widetilde{M}} \operatorname{Gr}_{k}^{\widetilde{W}^{\prime \prime}} \text { is an isomor phism for } l \geqq 1 .
$$

This follows from

$$
N_{1}^{l}: \operatorname{Gr}_{a}^{W} \mathrm{Gr}_{k+l}^{\tilde{M}} \mathrm{Gr}_{k}^{\tilde{W}^{\prime \prime}} \longrightarrow \mathrm{Gr}_{a}^{W} \mathrm{Gr}_{k-l}^{\tilde{\mathbb{M}}} \mathrm{Gr}_{k}^{\widetilde{W}^{\prime \prime}} \text { is an isomorphism. }
$$

By virtue of Lemma 5.5.3, $\mathrm{Gr}^{W} \mathrm{Gr}^{\tilde{M}} \mathrm{Gr}^{\tilde{W}}=\mathrm{Gr}^{\tilde{M}} \mathrm{Gr}^{\tilde{W}} \mathrm{Gr}^{W}$. Then, by $\mathbb{L e m m a}$ 3.4.2, when $k<a-1, \mathrm{Gr}_{a}^{W} \mathrm{Gr}_{k}^{W^{\prime \prime}}=0$. When $k=a-1, \mathrm{Gr}_{a}^{W} \mathrm{Gr}_{k}^{W^{\prime \prime}}=N_{2} \mathrm{Gr}_{a}^{W}$ and (5.5.5) follows from Proposition 5.4.2 along with Lemma 5.5.2. Trerefore we can assume $k \geqq a$. We shall show, in this case

$$
\tilde{M}\left(\mathrm{Gr}_{k}^{\tilde{W}} \mathrm{Gr}_{a}^{W}\right)=M\left(\mathrm{Gr}_{k}^{\tilde{W}} \mathrm{Gr}_{a}^{W}\right)
$$

Note that $\tilde{W}_{k}^{\prime \prime} \cap W_{a}=N_{2} W_{a}+M_{k}^{\prime \prime} \cap W_{a}$ and $\tilde{M}_{l} \cap W_{a}=N_{2}\left(M_{l+1}^{\prime} \cap W_{a}\right)+M_{l} \cap M_{l}^{\prime}$ $\cap W_{a}$ by Lemma 5.5.2. Since $N_{2} W_{a} \subset \widetilde{W}_{k-1}^{\prime \prime}$, we have

$$
\tilde{M}_{i}\left(\mathrm{Gr}_{k}^{\tilde{W}^{\prime \prime}} \mathrm{Gr}_{a}^{W}\right) \subset M_{l}\left(\mathrm{Gr}_{k}^{\tilde{W}^{\prime \prime}} \mathrm{Gr}_{a}^{W}\right)
$$

Conversely, (3.1.5) implies

$$
M_{l} \cap W_{a} \subset N_{2}\left(M_{l+2} \cap W_{a}\right)+M_{l} \cap M_{l}^{\prime} \cap W_{a} \subset \tilde{W}_{k-1}^{\prime \prime}+\tilde{M}_{l} \cap \mathbb{W}_{a}
$$

and hence $M\left(\mathrm{Gr}_{k}^{\widetilde{W}} \mathrm{Gr}_{a}^{W}\right) \subset \tilde{M}\left(\mathrm{Gr}_{k}^{W^{\prime \prime}} \mathrm{Gr}_{a}^{W}\right)$. Thus (5.5.5) follows from (5.5.7) $\quad N_{1}^{l}: \mathrm{Gr}_{k+l}^{M} \mathrm{Gr}_{k}^{W^{\prime \prime}} \mathrm{Gr}_{a}^{W} \leftrightharpoons \mathrm{Gr}_{k-l}^{M} \mathrm{Gr}_{k}^{W^{\prime \prime}} \mathrm{Gr}_{a}^{W} \quad$ for $k \geqq a, l \geqq 1$.

Since $\operatorname{Gr}_{k}^{\tilde{W}}$ " $\mathrm{Gr}_{a}^{W}$ is a direct summand of $\operatorname{Gr}_{k}^{\tilde{\tilde{T}}} \mathrm{Gr}_{a}^{W}$ by Lemma 3.4.2 and 
$M\left(\mathrm{Gr}_{a}^{W}\right)=M\left(N_{1} \mid \mathrm{Gr}_{a}^{W} ; M^{\prime \prime}\left(\mathrm{Gr}_{a}^{W}\right)\right)$, we have (5.5.7) and this completes the proof of Theorem 5.5.1.

As a corollary of Theorem 5.5.1 we have

Corollary 5.5.4. If $\left(W ; F, \bar{F} ; N_{1}, \ldots, N_{l}\right)$ is an IMHM, then so are $\left(N_{1 *} W ; F, \bar{F} ; N_{1}, \ldots, N_{l}\right)$ and $\left(M\left(N_{1}, W\right) ; F, \bar{F} ; N_{2}, \ldots, N_{l}\right)$.

Use Proposition 5.3.1

\section{Proposition 5.5.5.}

$$
\begin{aligned}
\left(N_{1 *} N_{2 *} W\right)_{k}=N_{1}\left(N_{2 *} W\right)_{k+1}+N_{2}\left(N_{1 *} W\right)_{k+1} & \\
& +M_{k}\left(N_{1}+N_{2}, W\right) \cap\left(N_{1 *} W\right)_{k} \cap\left(N_{2 *} W\right)_{k} \\
=N_{1}\left(N_{2 *} W\right)_{k+1}+N_{2}\left(N_{1 *} W\right)_{k+1} & \\
& +M_{k}\left(N_{1}+N_{2}, W\right) \cap\left(N_{1 *} W\right)_{k+1} \cap\left(N_{2 *} W\right)_{k+1}
\end{aligned}
$$

In particular $N_{1 *} N_{2 *} W=N_{2 *} N_{1 *} W$.

Proof. We shall use the notation in (5.5.1). Noting $\left(N_{1 *} N_{2 *} W\right)_{k}=$ $N_{1} \tilde{W}_{k+1}^{\prime \prime}+\tilde{M}_{k} \cap \widetilde{W}_{k}^{\prime \prime}=\left(N_{1} \widetilde{W}_{k+1}^{\prime \prime}+\tilde{M}_{k}\right) \cap \widetilde{W}_{k+1}^{\prime \prime}$, it is enough to prove

$$
\begin{aligned}
& \tilde{M}_{k} \cap \tilde{W}_{k}^{\prime \prime} \subset N_{2} \tilde{W}_{k+1}^{\prime}+M_{k} \cap \widetilde{W}_{k}^{\prime \prime} \cap \tilde{W}_{k}^{\prime}, \\
& N_{2} \tilde{W}_{k+1}^{\prime} \subset N_{1} \tilde{W}_{k+1}^{\prime \prime}+\tilde{M}_{k} \cap \tilde{W}_{k}^{\prime \prime},
\end{aligned}
$$

and

$$
M_{k} \cap \tilde{W}_{k+1}^{\prime} \subset N_{1} \tilde{W}_{k+1}^{\prime \prime}+\tilde{M}_{k}
$$

Note that

$$
\tilde{M}_{k}=N_{2} M_{k+1}^{\prime}+M_{k} \cap M_{k}^{\prime}
$$

By Lemma 5.5.2 we have

$$
\begin{aligned}
\tilde{M}_{k} \cap \tilde{W}_{k}^{\prime \prime} \subset \tilde{M}_{k} \cap W_{k+1} \subset N_{2}\left(M_{k+1}^{\prime} \cap W_{k+1}\right) & +M_{k} \cap M_{k}^{\prime} \cap W_{k+1} \\
& \subset N_{2} \tilde{W}_{k+1}^{\prime} \cap \tilde{W}_{k}^{\prime \prime}+M_{k} \cap \tilde{W}_{k}^{\prime} .
\end{aligned}
$$

Therefore we have (5.5.8).

Secondly, (5.5.9) follows from

$$
\begin{array}{r}
N_{2} \tilde{W}_{k+1}^{\prime}=N_{2}\left(N_{1} W_{k+2}+M_{k+1}^{\prime} \cap W_{k+1}\right) \subset N_{1} N_{2} W_{k+2}+N_{2} M_{k+1}^{\prime} \cap N_{2} W_{k+1} \\
\subset N_{1} \tilde{W}_{k+1}^{\prime \prime}+\tilde{M}_{k} \cap \tilde{W}_{k}^{\prime \prime} .
\end{array}
$$

Finally, by (3.1.5), we have $M_{k} \cap \tilde{W}_{k+1}^{\prime}=M_{k} \cap\left(N_{1} W_{k+2}+M_{k+1}^{\prime} \cap W_{k+1}\right) \subset N_{1}\left(M_{k+2} \cap W_{k+2}\right)+M_{k} \cap M_{k+1}^{\prime}$ 


$$
\begin{aligned}
& \subset N_{1}\left(N_{2} W_{k+2}+M_{k+2} \cap M_{k+2}^{\prime} \cap W_{k+2}\right)+M_{k} \cap M_{k+1}^{\prime} \\
& \subset N_{1} N_{2} W_{k+2}+M_{k} \cap M_{k+1}^{\prime} \subset N_{1} \tilde{W}_{k+1}^{\prime \prime}+\tilde{M}_{k} .
\end{aligned}
$$

5.6. Let $I$ be a finite set and let $S$ be the set of subsets of $I$. Let us consider the following category $\mathbb{M H}(I)$ consisting of $\mathbb{H}=\left\{\mathbb{H}(\alpha), W^{\alpha}(\mathbb{H}), F_{\alpha}(\mathbb{H}), \bar{F}_{\alpha}(\mathbb{H})\right.$, $\left.f_{\alpha \beta}(H), g_{\beta \alpha}(H)\right\}_{\beta \subset \alpha \subset I}$. Here, $\mathbb{H}(\alpha)$ is a finite-dimensional vector space, $F_{\alpha}(\mathbb{H})$, $\bar{F}_{\alpha}(\mathrm{H})$ and $W^{\alpha}(\mathrm{H})$ are filtrations of $\mathrm{H}(\alpha)$ and $f_{\alpha \beta}(\mathrm{H}): \mathrm{H}(\beta) \rightarrow \mathrm{H}(\alpha), g_{\beta \alpha}(\mathrm{H}): \mathbb{H}(\alpha)$ $\rightarrow \mathrm{H}(\beta)$ satisfy the following conditions (5.6.1)-(5.6.7):

(5.6.1) $f_{\alpha \alpha}=g_{\alpha \alpha}=i d$,

(5.6.2) $f_{\alpha \beta} \circ f_{\beta \gamma}=f_{\alpha \gamma}, \quad g_{\gamma \beta} \circ g_{\beta \alpha}=g_{\gamma \alpha} \quad$ for $\alpha \supset \beta \supset \gamma$.

(5.6.3) $g_{\alpha, \alpha \cup \beta} f_{\alpha \cup \beta, \beta}=f_{\alpha, \alpha \cap \beta} g_{\alpha \cap \beta, \beta} \quad$ for any $\alpha$ and $\beta$.

(5.6.4) $f_{\alpha \beta} F_{\beta}^{p}(\mathrm{H}) \subset F_{\alpha}^{p-|\alpha| \beta \mid}(\mathrm{H}), \quad f_{\alpha \beta} \bar{F}_{\beta}^{p}(\mathrm{H}) \subset \bar{F}_{\alpha}^{p-|\alpha| \beta \mid}(\mathrm{H})$,

$f_{\alpha \beta} W_{k}^{\beta}(\mathrm{H}) \subset W_{k-|\alpha| \beta \mid}^{\alpha}(\mathrm{H}), g_{\beta \alpha} F_{\alpha}^{p}(\mathrm{H}) \subset F_{\beta}^{p}(\mathbb{H})$, $g_{\beta \alpha} \bar{F}_{\alpha}^{p}(\mathrm{H}) \subset \bar{F}_{\beta}^{p}(\mathrm{H})$ and $g_{\beta \alpha} W_{k}^{\alpha}(\mathrm{H}) \subset W_{k+|\alpha| \beta \mid}^{\beta}(\mathrm{H}) \quad$ for $\quad \beta \subset \alpha \subset \mathbb{I}$.

(5.6.5) Letting $N_{j} \in \operatorname{End}(\mathbb{H}(\alpha))$ be $N_{j}=g_{\alpha, \alpha \cup\{j\}} f_{\alpha \cup\{j\}, \alpha} \quad$ for $a \ngtr j$ and $N_{j}=f_{\alpha, \alpha \backslash\{j\}} g_{\alpha \backslash\{j\}, j} \quad$ for $a \ni j, \quad N_{j}$ is nilpotent .

(5.6.6) $\left\{\mathbb{H}(\alpha) ; W^{\alpha}(\mathrm{H}) ; F_{\alpha}(\mathrm{H}), \bar{F}_{\alpha}(\mathbb{H}) ;\left\{N_{j}\right\}_{j \in I}\right\}$ is an IMHM for any $\alpha \subset \mathbb{I}$.

(5.6.7) $\mathrm{Gr}_{k}^{W \alpha} \rightleftarrows \mathrm{Gr}_{k-|\alpha| \beta \mid}^{W \beta} \quad$ for $\alpha \supset \beta$.

Note that if $\mathrm{H}$ is in $\mathrm{MH}(I)$, then $H^{*}$ defined as follows is also an object of $\mathrm{MH}(\mathbb{I})$

$$
\begin{aligned}
& H^{*}(\alpha)=H(\alpha)^{*} \\
& W_{k}^{\alpha}\left(\mathbb{H}^{*}\right)=\left(\mathbb{H}(\alpha) / W_{-k+2|\alpha|-1}^{\alpha}(\mathbb{H})\right)^{*}, \\
& F_{\alpha}^{p}\left(\mathbb{H}^{*}\right)=\left(\mathbb{H}(\alpha) / F_{\alpha}^{1-p+|\alpha|}(\mathbb{H})\right)^{*}, \\
& \bar{F}_{\alpha}^{p}\left(\mathbb{H}^{*}\right)=\left(\mathbb{H}(x) / \bar{F}_{\alpha}^{1-p+|\alpha|}(\mathbb{H})\right)^{*}, \\
& f_{\alpha \beta}\left(\mathbb{H}^{*}\right)=g_{\beta \alpha}(\mathbb{H})^{*} \\
& g_{\beta \alpha}\left(\mathbb{H}^{*}\right)=(-1)^{|\beta|-|\alpha|} f_{\alpha \beta}(\mathbb{H})^{*}
\end{aligned}
$$

For $\mathbb{H} \in \mathbb{M H}(I)$, let us define the Tate twist $\mathbb{H}(n)$ of $\mathbb{H}$ by

$$
\begin{aligned}
& \mathrm{H}(n)(\alpha)=\mathbb{H}(\alpha), \quad W_{k}^{\alpha}(\mathbb{H}(n))=W_{k+2 n}^{\alpha}(\mathbb{H}), \\
& F_{\alpha}^{p}(\mathbb{H}(n))=F^{p+n}(\mathbb{H}), \quad \bar{F}_{\alpha}^{p}(\mathbb{H}(n))=\bar{F}_{\alpha}^{p+n}(\mathbb{H}), \\
& f_{\alpha \beta}(\mathbb{H}(n))=f_{\alpha \beta}(\mathbb{H}), \quad g_{\beta \alpha}(H(n))=g_{\beta \alpha}(\mathbb{H}) .
\end{aligned}
$$

Lemma 5.6.1. If $\alpha \subset \beta$, and $\gamma \supset \beta \mid \alpha$,

$$
\begin{aligned}
& f_{\beta \alpha}(\mathbb{H}) M_{k}(\gamma, \mathbb{H}(\alpha)) \subset M_{k-2|\beta| \alpha \mid}(\gamma, \mathbb{H}(\beta)), \\
& g_{\alpha \beta}(\mathbb{H}) M_{k}(\gamma, H(\alpha)) \subset M_{k}(\gamma, \mathbb{H}(\beta)) .
\end{aligned}
$$


Here, $M(\gamma, \mathrm{H}(\alpha))=M\left(N, W^{\alpha}(\mathrm{H})\right)$ for $N \in \mathrm{C}(\gamma)$.

Proof. We shall prove them by the induction of $|\beta| \alpha \mid$. If $|\beta| \alpha \mid=1$ and $\gamma=\beta \backslash \alpha$, they follow from Proposition 3.3.1. When $|\beta| \alpha \mid=1$, they follow from this case and the fact that $M(\gamma, \mathrm{H}(\alpha))=M(N, M(\beta \mid \alpha, \mathrm{H}(\alpha)))$ for $N \in \mathrm{C}(\gamma)$. Now assume $|\beta| \alpha \mid>1$. Then taking $\delta$ such that $\alpha \subsetneq \delta \subsetneq \beta$, they follow from the hypothesis of the inductions for $\alpha, \delta$ and $\delta, \beta$.

Lemma 5.6.2. If we set

$$
P_{k}^{\alpha}(\mathrm{H})=\underset{\beta_{\mp}^{-\alpha}}{\cap} \operatorname{Ker}\left(g_{\beta, \alpha}: \mathrm{Gr}_{k}^{W \alpha(\mathrm{H})} \longrightarrow \mathrm{Gr}_{k+|\alpha| \beta \mid}^{W \beta(\mathrm{H})}\right) \subset \mathrm{Gr}_{k}^{W^{\alpha}(\mathrm{H})}
$$

then $P_{k}^{\alpha}(\mathrm{H})$ has weight $k$ with respect to the weight filtration $M(\alpha, \mathbb{H}(\alpha))$.

Proof. We may assume that $\alpha=I$. Take $N \in \mathrm{C}(I)$. Then $M(\alpha, \mathrm{H}(\alpha))$ induces the weight filtration of $N \mid \mathrm{Gr}_{k}^{W^{\alpha}(\mathrm{H})}$, shifted by $k$. On ther other hand, $P_{k}^{\alpha}(\mathrm{H})$ is a direct summand of $\mathrm{Gr}_{k}^{W \alpha(\mathrm{H})}$ as an $\mathbb{C}\left[N_{1}, \ldots, N_{l}\right]$-module by Proposition 3.3.1. Therefore $M(\alpha, \mathrm{H}(\alpha))$ induces on $P_{k}^{\alpha}(\mathrm{H})$ the weight filtration of $N \mid P_{k}^{\alpha}(\mathrm{H})$ shifted by $k$. Then this Lemma follows from $N \mid P\left(\mathbb{H}_{k}^{\alpha}\right)=0$. Q.E.D.

For $\gamma \in I$, we define $\psi_{\gamma}(\mathrm{H})$ and $\varphi_{\gamma}(\mathrm{H})$ in $\mathrm{MH}(I \mid \gamma)$ as follow

$$
\begin{aligned}
& \psi_{\gamma}(\mathrm{H})(\alpha)=\mathrm{H}(\alpha), \quad W^{\alpha}\left(\psi_{\gamma}(\mathrm{H})\right)=M(\gamma, \mathrm{H}(\alpha)), \\
& F_{\alpha}\left(\psi_{\gamma}(\mathrm{H})\right)=F_{\alpha}(\mathrm{H}), \quad \bar{F}_{\alpha}\left(\psi_{\gamma}(\mathrm{H})\right)=\bar{F}_{\alpha}(\mathrm{H}), \\
& f_{\alpha \beta}\left(\psi_{\gamma}(\mathrm{H})\right)=f_{\alpha \beta}(\mathrm{H}) \text { and } g_{\beta \alpha}\left(\psi_{\gamma}(\mathrm{H})\right)=g_{\beta \alpha}(\mathrm{H}) \quad \text { for } \quad \alpha \subset \beta \subset I \mid \gamma \\
& \text { (5.6.12) } \quad \varphi_{\gamma}(\mathrm{H})(\alpha)=\mathrm{H}(\alpha \cup \gamma), \quad W^{\alpha}\left(\psi_{\gamma}(\mathrm{H})\right)=M(\gamma, \mathrm{H}(\alpha \cup \gamma)) \\
& F_{\alpha}\left(\varphi_{\gamma}(\mathrm{H})\right)=F_{\alpha \cup \gamma}(\mathrm{H}), \quad \bar{F}_{\alpha}\left(\varphi_{\gamma}(\mathrm{H})\right)=\bar{F}_{\alpha \cup \gamma}(\mathrm{H}) \\
& f_{\alpha \beta}\left(\varphi_{\gamma}(\mathrm{H})\right)=f_{\alpha \cup \gamma, \beta \cup \gamma}(\mathrm{H}) \text { and } \\
& g_{\beta \gamma}\left(\varphi_{a}(\mathrm{H})\right)=g_{\beta \cup \gamma, \alpha \cup \gamma}(\mathrm{H}) \quad \text { for } \quad \alpha \subset \beta \subset I \mid \gamma \text {. }
\end{aligned}
$$

Proposition 5.6.4. $\psi_{\gamma}(\mathrm{H})$ and $\varphi_{\gamma}(\mathrm{H})$ are objects in $\mathrm{MH}(I \backslash \gamma)$.

Proof. (5.6.1)-(5.6.5) are evident and (5.6.6) follows from Proposition 5.3.1.

In order to prove (5.6.7), we prepare the following

Lemma 5.6.5. Let $\left(\mathrm{H} ; F, \bar{F} ; N_{1}, N_{2}\right)$ and $\left(\mathrm{H}^{\prime} ; F^{\prime}, \bar{F}^{\prime} ; N_{1}^{\prime}, N_{2}^{\prime}\right)$ be a nilpotent orbit of weight $w$ and $w-1$, respectively. Let $f: H \rightarrow \mathbb{H}^{\prime}$ and $g: \mathbb{H}^{\prime} \rightarrow \mathbb{H}$ be homomorphisms such that

$$
\begin{aligned}
& g \circ f=N_{1}, \quad f \circ g=N_{1}^{\prime}, \\
& N_{2}^{\prime} \circ f=f \circ N_{2}, \quad N_{2} \circ g=g \circ N_{2}^{\prime},
\end{aligned}
$$




$$
H \underset{\substack{g}}{\stackrel{f}{\rightleftarrows}} \mathbb{H}^{\prime} .
$$

Then, we have for any $k$

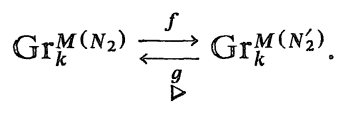

Here $M(*)$ is the weight filtration.

Proof. By the duality, it is enough to show

$$
g M_{k}\left(N_{2}^{\prime}\right) \subset N_{1} M_{k}\left(N_{2}\right)+M_{k-1}\left(N_{2}\right) .
$$

We have, by Proposition 5.4.2,

$$
g M_{k}\left(N_{2}^{\prime}\right) \subset M_{k}\left(N_{2} \mid \operatorname{Im} g\right)=M_{k}\left(N_{2} \mid \operatorname{Im} N_{1}\right) \subset N_{1} M_{k}\left(N_{2}\right)+M_{k-1}\left(N_{2}\right) .
$$

Now, we shall show (5.6.7) for $\psi_{\gamma}(\mathrm{H})$ and $\varphi_{\gamma}(\mathrm{H})$. For this, it is enough to show, by Proposition 2.3.1

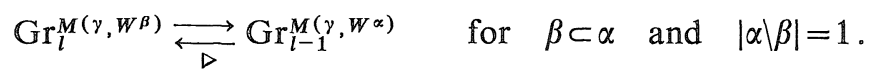

By Theorem 3.2.9, $\operatorname{Gr}_{l}^{M\left(\gamma, W^{\beta}\right)}$ is canonically a direct sum of $\operatorname{Gr}_{l}^{M\left(\gamma, W^{\beta}\right)} \operatorname{Gr}_{k}^{W \beta}$ s. Hence (5.6.19) follows from

$$
\mathrm{Gr}_{l}^{M\left(\gamma, W^{\beta}\right)} \mathrm{Gr}_{k}^{W^{\beta}} \underset{\triangleright}{\rightleftarrows} \operatorname{Gr}_{l-1}^{M\left(\gamma, W^{\beta}\right)} \mathrm{Gr}_{k-1}^{W^{\beta}}
$$

which is an immediate consequence of Lemma 5.6.5.

5.7. By Lemma 5.6.1, $f_{\alpha \beta}(\mathbb{H})$ and $g_{\beta \alpha}(\mathbb{H})$ are considered as morphisms of

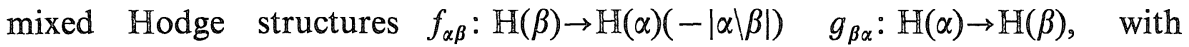
$M(I, H(\alpha))$ as weight filtrations.

Letting $e_{j}(j \in \mathbb{I})$ be the base of $\mathbb{Z}^{(I)}$, we shall define the complex $\mathbb{I}(\mathbb{H})$ by

$$
\Pi(\mathbb{H})^{n}=\underset{|\alpha|=n}{\oplus} \mathbb{H}(\alpha)(-n) \underset{\mathbb{Z}}{\otimes} \mathbb{Z} e_{\alpha} .
$$

Here $e_{\alpha}=\underset{j \in \alpha}{\wedge} e_{j}$. The differential is given by $\sum_{j, \alpha} f_{\alpha \cup\{j\}, \alpha} e_{j}$. Let us define $W_{k}(\Pi(\mathbb{H}))$ by

$$
W_{k}\left(\Pi(\mathbb{H})^{n}\right)=\underset{|\alpha|=n}{\oplus} W_{k-n}(\mathbb{H}(\alpha))(-n) \otimes e_{\alpha} .
$$

Then $\Pi(\mathrm{H})$ is a filtrered complex in the category of mixed Hodge structures. 
Theorem 5.7.1. $\mathrm{H}^{n}\left(W_{k}(\Pi(\mathrm{H}))\right)$ has weight $\leqq k+n$.

Proof. It is enough to show that $\mathbb{H}^{n}\left(\mathrm{Gr}_{a}^{W} \Pi(\mathrm{H})\right)$ has weight $\leqq a+n$. Then replacing $\mathrm{H}$ with $\mathrm{Gr}^{W}$ we may assume

$$
W_{k}^{\alpha}(\mathrm{H})= \begin{cases}H(\alpha) & k=a-|\alpha| \\ 0 & k \neq a-|\alpha| .\end{cases}
$$

Then Proposition 2.3.1 implies that $\mathrm{H}$ is a direct sum of $M$ (I, (vector spaces)). Thus, we may assume that, there is $\gamma \subset I$ such that $H(\alpha)=0$ if $\alpha \not \supset \gamma$ and $H(\alpha)$ $=\left(\prod_{j \in \gamma \mid \alpha} N_{j}\right) \mathrm{H}(\gamma)$ for $\alpha \supset \gamma$. In this case, the theorem is nothing but the purity theorem $([\mathrm{K}-\mathrm{K}],[\mathrm{C}-\mathrm{K}-\mathrm{S}])$.

5.8. Let $\mathrm{H}=\left(W ; F, \bar{F} ; N_{1}, \ldots, N_{l}\right)$ be an IMHM. For $J \subset I=\{1, \ldots, l\}$, we set

$$
\begin{aligned}
& M(J)=M(J ; W)=M\left(\sum_{j \in J} N_{j}, W\right), \\
& \Psi_{J} W=N_{j_{1} *} \cdots N_{j_{r} *} W \text { where } J=\left\{j_{1}, \ldots, j_{r}\right\} .
\end{aligned}
$$

By Corollary 5.5.4 and Proposition 5.5.5 $\Psi_{J} W$ is well-defined.

Set

$$
\mathrm{C}(J)=\left\{\sum_{j \in J} t_{j} N_{j} ; t_{j}>0\right\} .
$$

Then we have already remarked in Proposition 5.2.5.

$$
\begin{aligned}
& M\left(J_{1} \cup J_{2}\right)=M\left(N, M\left(J_{2}\right)\right) \quad \text { for } \quad N \in \mathbb{C}\left(J_{1}\right) \text {, } \\
& \text { or } \\
& M\left(J_{1} \cup J_{2}\right)=M\left(J_{1}, M\left(J_{2}\right)\right) .
\end{aligned}
$$

Applying Theorem 5.5.1, we have

$$
M\left(J_{1}, \Psi_{J_{2}} W\right)=\Psi_{J_{2}} M\left(J_{1}, W\right) .
$$

We have also

$$
M\left(K, \Psi_{J} W\right)=M(K, W) \quad \text { for } \quad K \supset J
$$

In fact, for $K \ni j$, we have $N_{j} M_{k}(K, W) \subset M_{k-2}(K, W)$, which implies $\left(N_{j}\right)_{*} M(K$, $W)=M(K, W)$.

By Proposition 2.3.1 and Lemma 3.4.2, we can associate to $W$ the object $D I(\mathrm{H})$ of $\mathrm{MH}(I)$ given by $D I(\mathrm{H})(\alpha)=\mathrm{H}, W^{\alpha}(D I(\mathrm{H}))=\Psi_{\alpha} W, F_{\alpha}(D I(\mathrm{H}))=F$, $\bar{F}_{\alpha}(D I(\mathrm{H}))=\bar{F}, f_{\alpha \beta}(D I(\mathrm{H}))=\prod_{j \in \alpha \backslash \beta} N_{j}, g_{\beta \alpha}(D I(\mathrm{H}))=i d$. 
Proposition 5.8.1. Let $\mathbb{H}^{\prime}=\left(W ; F, \bar{F} ; N_{1}, \ldots, N_{l}\right)$ be an IMHS and $H \in \mathrm{MH}(I)$. Then we have

$$
\operatorname{Hom}_{\mathrm{MH}(I)}\left(\mathbb{H}, D I\left(\mathbb{H}^{\prime}\right)\right)=\mathbb{H o m}\left(\mathbb{H}(\phi), \mathbb{H}^{\prime}\right) \text {. }
$$

Here the last $\mathrm{Hom}$ is the sei of homomorphisms preserving $W, F, \bar{F}$ and commuting with $N_{1}, \ldots, N_{l}$.

Proof. It is enough to show that $g_{\phi \beta} W^{\beta}(\mathbb{H}) \subset \Psi_{\beta} W^{\phi}(\mathbb{H})$. We shall prove

$$
g_{\beta \alpha} W^{\alpha}(\mathrm{H}) \subset \Psi_{\alpha \mid \beta} W^{\beta}(\mathbb{H}) \quad \text { for } \quad \beta \subset \alpha,
$$

by the induction of $|\alpha| \beta \mid$. If $\alpha=\beta$, there is nothing to prove. Assume $|\alpha| \beta \mid=1$. Set $\alpha \backslash \beta=\{j\}$. Then by (5.6.7)

$$
\begin{aligned}
g_{\beta \alpha} W_{k}^{\alpha}(\mathbb{H})= & N_{j} W_{k+1}^{\beta}(\mathrm{H})+g_{\beta \alpha} W_{k}^{\alpha}(\mathbb{H}) \cap W_{k}^{\beta}(\mathbb{H}) \\
& \subset N_{j} W_{k+1}^{\beta}(\mathbb{H})+g_{\beta \alpha}\left(M_{k}\left(N_{j}, W^{\alpha}(\mathbb{H})\right) \cap W_{k}^{\alpha}(\mathbb{H})+W_{k-1}^{\alpha}(\mathbb{H})\right) \\
& \subset N_{j} W_{k+1}^{\beta}(\mathbb{H})+M_{k}\left(N_{j}, W^{\beta}(\mathbb{H})\right) \cap W_{k+1}^{\beta}(\mathbb{H})+g_{\beta \alpha} W_{k-1}^{\alpha}(\mathbb{H}) \\
= & \left(N_{j *} W^{\beta}(\mathbb{H})\right)_{k}+g_{\beta \alpha} W_{k-1}^{\alpha}(\mathbb{H}) .
\end{aligned}
$$

Here the last inclusion follows from Proposition 3.3.1.

Now assume $|\alpha \backslash \beta| \geqq 2$. Take $\gamma$ such that $\beta \varsubsetneqq \gamma \varsubsetneqq \alpha$. Then the induction on $|\alpha| \beta \mid$ shows

Therefore we have

$$
\begin{aligned}
& g_{\gamma \alpha} W^{\alpha}(\mathrm{H}) \subset \Psi_{\alpha \backslash \gamma} W^{\gamma}(\mathbb{H}) \\
& g_{\beta \gamma} W^{\gamma}(\mathbb{H}) \subset \Psi_{\gamma \backslash \beta} W^{\beta}(\mathbb{H}) .
\end{aligned}
$$

$$
\begin{aligned}
g_{\beta \alpha} W^{\alpha}(\mathbb{H}) \subset g_{\beta \gamma} \Psi_{\alpha \backslash \gamma} W^{\gamma}(\mathbb{H}) & \\
& \subset \Psi_{\alpha, \gamma} g_{\beta \gamma} W^{\gamma}(\mathbb{H}) \subset \Psi_{\alpha \mid \gamma} \Psi_{\gamma \backslash \beta} W^{\beta}(\mathbb{H})=\Psi_{\alpha \backslash \beta} W^{\beta}(\mathbb{H}) .
\end{aligned}
$$

Proposition 5.8.2.

$$
\begin{aligned}
\left(\Psi_{J} W\right)_{h} & =\sum_{j \in J} N_{j}\left(\Psi_{J \backslash\{j\}} W\right)_{k+1}+M_{k}(J) \cap \bigcap_{j \in J}\left(\Psi_{J \backslash\{j\}} W\right)_{k+1} \\
& =\sum_{j \in J} N_{j}\left(\Psi_{J \backslash\{j\}} W\right)_{k+1}+M_{k}(J) \cap \bigcap_{j \in J}\left(\Psi_{J \backslash\{j\}} W\right)_{k} .
\end{aligned}
$$

Proof. We shall prove this by the induction of $|J|$. For $j \in J$, we have

$$
M(J) \cap\left(\Psi_{J \backslash\{j\}} W\right)_{k+1}=M\left(N_{j}, M(J \backslash\{j\}) \cap\left(\Psi_{J \backslash\{j\}} W\right)_{k+1}\right)
$$

because $M(J \backslash\{j\})=M\left(J \backslash\{j\}, \Psi_{J \backslash\{j\}} W\right)$. Therefore we have

$$
\begin{aligned}
& M_{k}(J) \cap\left(\Psi_{J \backslash\{j\}} W\right)_{k+1} \\
& \quad \subset N_{j}\left(M_{k+2}(J) \cap\left(\Psi_{J \backslash\{j\}} W\right)_{k+1}\right) \\
& \quad+M_{k}(J) \cap M_{k}(J \backslash\{j\}) \cap\left(\Psi_{J \backslash\{j\}} W\right)_{k+1}
\end{aligned}
$$


Note that

$$
N_{j} \Psi_{J \backslash\{j\}} W \subset \Psi_{J \backslash\{i\}} W \quad \text { for any } \quad i, j \in J .
$$

In fact, if $i \neq j, N_{j}\left(\Psi_{J \backslash\{j\}} W\right)_{k} \subset N_{j}\left(\Psi_{J \backslash\{j, i\}}(W)\right)_{k+1} \subset\left(\Psi_{J \backslash\{i\}} W\right)_{k}$. Then (5.8.8) and (5.8.9) imply

$$
\begin{aligned}
M_{k}(J) \cap & \bigcap_{i \in J}\left(\Psi_{J \backslash\{i\}} W\right)_{k+1} \\
& \subset N_{j}\left(\Psi_{J \backslash\{j\}} W\right)_{k+1}+M_{k}(J) \cap M_{k}(J \backslash\{j\}) \cap \bigcap_{i \in J \backslash\{j\}}\left(\Psi_{J \backslash\{i\}} W\right)_{k+1} .
\end{aligned}
$$

We have

$$
\begin{aligned}
M_{k}(J) \cap M_{k}(J \backslash\{j\})=M_{k}\left(N_{j}, M(J \backslash\{j\})\right) & \cap M_{k}(J \backslash\{j\}) \\
& \subset\left(N_{j *} M(J \backslash\{j\})\right)_{k}=M\left(J \backslash\{j\}, \Psi_{\{j\}} W\right)_{k} .
\end{aligned}
$$

Therefore by the induction hypothesis,

$$
\begin{aligned}
M_{k}(J) & \cap M_{k}(J \backslash\{j\}) \cap \underset{i \in J \backslash\{j\}}{\cap}\left(\Psi_{J \backslash\{i\}} W\right)_{k+1} \\
& \subset M_{k}\left(J \backslash\{j\}, \Psi_{\{j\}} W\right) \cap \bigcap_{i \in J \backslash\{j\}}\left(\Psi_{J \backslash\{j\} \backslash\{i\}} \Psi_{\{j\}} W\right)_{k+1} \\
& \subset\left(\Psi_{J \backslash\{j\}} \Psi_{\{j\}} W\right)_{k}=\left(\Psi_{J} W\right)_{k} .
\end{aligned}
$$

This shows that

$$
\sum_{j \in J} N_{j}\left(\Psi_{J \backslash\{j\}} W\right)_{k+1}+M_{k}(J) \cap \bigcap_{j \in J}\left(\Psi_{J \backslash\{j\}} W\right)_{k+1} \subset\left(\Psi_{J} W\right)_{k} .
$$

On the other hand, since $D I(\mathrm{H})$ belongs to $\mathrm{MH}(I)$, we can apply Lemma 5.6.2 and we obtain

$$
\begin{aligned}
\operatorname{Gr}_{k}^{\Psi_{J}(W)}=\sum \operatorname{Im}\left(\mathrm{Gr}_{k+1}^{\Psi_{j}(j) W}\right. & \left.\longrightarrow \operatorname{Gr}_{k}^{\Psi_{J} W}\right) \\
& +M_{k}(J) \cap \bigcap_{j \in J} \operatorname{Ker}\left(\operatorname{Gr}_{k}^{\Psi_{J} W} \longrightarrow \operatorname{Gr}_{k+1}^{\Psi_{J}(j) W}\right),
\end{aligned}
$$

or

$$
\begin{aligned}
\left(\Psi_{J} W\right)_{k} \subset \sum_{j \in J} N_{j}\left(\Psi_{J \backslash\{j\}} W\right)_{k+1} & \\
& +M_{k}(J) \cap \bigcap_{j \in J}\left(\Psi_{J \backslash\{j\}} W\right)_{k}+\left(\Psi_{J} W\right)_{k-1}
\end{aligned}
$$

Then the induction in $k$ shows that $\left(\Psi_{J} W\right)_{k}$ is contained in

$$
\sum_{j \in J} N_{j}\left(\Psi_{J \backslash\{j\}} W\right)_{k+1}+M_{k}(J) \cap \bigcap_{j \in J}\left(\Psi_{J\{\backslash j\}} W\right)_{k}
$$

§6. The Proof of Theorem 4.4 .1 .

6.1. In order to prove Theorem 4.4.1, it is enough to show the following. 
Lemma 6.1.1. Let $\mathbb{H}=\left(W ; F, \bar{F} ; N_{1}, N_{2}\right)$ be a pre-IMHM and assume that there exist the weight filtration of $N_{1}$ relative to $W$ and that of $N_{2}$. Then there exists the weight filtration $M$ of $N_{1}+N_{2}$ relative to $W$ and we have $N_{j} M_{k}$ $\subset M_{k-2}$ for $j=1,2$.

6.2. We shall show this by the induction. Hence, we may assume that $\mathbb{H}=W_{0}$ and that $\mathbb{H}$ induces on $W_{-1}$ an IMHM. Let $\mathbb{H}_{0}$ be $\mathrm{Gr}_{0}^{W}$. Then we have an exact sequence

$$
0 \longrightarrow W_{-1} \stackrel{\psi}{\longrightarrow} \mathbb{H} \stackrel{\varphi}{\longrightarrow} \mathbb{H}_{0} \longrightarrow 0 \text {. }
$$

Let $M\left(\mathrm{H}_{0}\right)$ and $M\left(W_{-1}\right)$ be the relative weight filtration of $N_{1}+N_{2}$ on $\mathbb{H}_{0}$ and $W_{-1}$, respectively. In order to prove 6.1 .1 , it is enough to show that there exists a section $s: \mathbb{H}_{0} \rightarrow \mathbb{H}$ of $\varphi$ in (6.2.1) satisfying the following condition (6.2.2). Note first that $\left[N_{j}, s\right]: \mathbb{H}_{0} \rightarrow \mathrm{H}$ splits as the composition of $\alpha_{j}(s): \mathbb{H}_{0} \rightarrow W_{-1}$ and $\psi$.

$$
\alpha_{j}(s) M_{k}\left(H_{0}\right) \subset M_{k-2}\left(W_{-1}\right) .
$$

In fact, if there exists such a section $s$ that satisfies (6.2.2),

$$
M_{k}=\psi\left(M_{k}\left(W_{-1}\right)\right)+s\left(M_{k}\left(\mathrm{H}_{0}\right)\right) \text { satisfies } \quad N_{j} M_{k} \subset M_{k-2},
$$

and it is evident that $M$ induces on $\mathrm{H}_{0}$ the weight filtration of $N_{1}+N_{2}$, shifted by $k$.

6.3. Now, we shall show the existence of such a section $s$. Set $\tilde{\mathrm{H}}=\operatorname{Hom}\left(\mathbb{H}_{0}, W_{-1}\right)$. Then $\tilde{\mathrm{H}}$ is an $\mathbb{I M H M}$. Then (6.2.2) is equivalent to $\alpha_{j}(s)$ $\in M_{-2}(\tilde{H})$. Let $M^{\prime}\left(\mathbb{H}_{0}\right), M^{\prime}(\mathbb{H}), M^{\prime}\left(W_{-1}\right)$ and $M^{\prime}(\tilde{H})$ be the relative weight filtration of $N_{1}$ on $\mathrm{H}_{0}, \mathbb{H}, W_{-1}$ and $\tilde{\mathrm{H}}$, respectively and let $M^{\prime \prime}\left(\mathbb{H}_{0}\right), M^{\prime \prime}(\mathbb{H})$, $M^{\prime \prime}\left(W_{-1}\right)$ and $M^{\prime \prime}(\tilde{\mathrm{H}})$ be the relative weight filtration of $N_{2}$. Since the exact sequence (6.2.1) is strict with respect to the filtration $\mathbb{M}^{\prime}$, there exists a section $s^{\prime}: H_{0} \rightarrow H$ such that $s^{\prime} M_{k}^{\prime}\left(H_{0}\right) \subset M_{k}^{\prime}(H)$. Therefore, we have

$$
\alpha_{1}\left(s^{\prime}\right) \in M_{-2}^{\prime}(\tilde{\mathbb{H}}) .
$$

Similarly if we take a section $s^{\prime \prime}: \mathbb{H}_{0} \rightarrow \mathbb{H}$ such that $s^{\prime \prime} M_{k}^{\prime \prime}\left(\mathbb{H}_{0}\right) \subset M_{k}^{\prime \prime}(\mathbb{H})$ we have

$$
\alpha_{2}\left(s^{\prime \prime}\right) \in M_{-2}^{\prime \prime}(\tilde{\mathrm{H}})
$$

By the assumption $t=s^{\prime}-s^{\prime \prime} \in \tilde{H}$. Note that $\tilde{\mathbb{H}}=W_{-1}(\tilde{H})$. Thus we have 


$$
\begin{aligned}
\alpha_{1}\left(s^{\prime}\right) \in & W_{-1}(\tilde{\mathrm{H}}) \cap M_{-2}^{\prime}(\tilde{\mathrm{H}}) \\
& \subset\left(N_{1 *} W(\tilde{\mathrm{H}})\right)_{-2}
\end{aligned}
$$

and $\alpha_{2}\left(s^{\prime}\right)=N_{2} t+\alpha_{2}\left(s^{\prime \prime}\right) \in N_{2} W_{-1}(\tilde{\mathrm{H}})+M_{-2}^{\prime \prime}(\tilde{\mathrm{H}}) \cap W_{-1}(\tilde{\mathrm{H}})=\left(N_{2 *} W(\tilde{\mathrm{H}})\right)_{-2}$.

Now, we consider the complex

$$
\begin{aligned}
W_{-1}(I I): W_{-1}(\tilde{\mathrm{H}}) & \longrightarrow\left(N_{1 *} W(\tilde{\mathrm{H}})\right)_{-2} \oplus\left(N_{2 *} W(\tilde{\mathrm{H}})\right)_{-2} \\
& \longrightarrow\left(N_{1 *} N_{2 *} W(\tilde{\mathrm{H}})\right)_{-3} .
\end{aligned}
$$

Then $\left(\alpha_{1}\left(s^{\prime}\right), \alpha_{2}\left(s^{\prime}\right)\right)$ is a cochain of $W_{-1}(\Pi)$ of degree $1 . \quad$ By the purity theorem for IMHM (Theorem 5.7.1) and the results in $\S 5.8, \mathbb{H}^{1}\left(W_{-1}(\Pi)\right.$ ) has weight $\leqq 0$, and hence the cohomology class of $\left(\alpha_{1}\left(s^{\prime}\right), \alpha_{2}\left(s^{\prime}\right)\right)$ is represented by a cochain of weight $\leqq 0$. Thus, there exists $u \in \tilde{H}$ such that

$$
\begin{aligned}
& \alpha_{1}\left(s^{\prime}\right)-N_{1} u \in M_{-2}(\tilde{\mathrm{H}}), \\
& \alpha_{2}\left(s^{\prime}\right)-N_{2} u \in M_{-2}(\tilde{\mathrm{H}}) .
\end{aligned}
$$

Then $s=s^{\prime}-u$ satisfies $\alpha_{j}(s) \in M_{-2}(\tilde{\mathrm{H}})$. This completes the proof of Theorem 4.4.1.

\section{References}

[C-K] E. Cattani and A. Kaplan, Polarized mixed Hodge structures and the local monodromy of a variation of Hodge structure, Inv. Math., 67 (1982), 101-115.

[C-K-S] E. Cattani, A. Kaplan and W. Schmid, 1. Degeneration of Hodge structures, preprint. 2. $L_{2}$ and intersection cohomologies for a polarizable variation of Hodge structures, preprint.

[D] P. Deligne, Théorie de Hodge I, II, III, Actes Congrès Intern. math. 1970, 425-430; Publ. Math. I. H. E. S., 40 (1971), 5-58; 44 (1974), 5-77.

[G-G-M] A. Galligo, M. Granger and Ph. Maisonobe, $D$-modules et faisceaux pervers dont le support singulier est un croisement normal, to appear in Ann. Sci. Éc. Norm. Sup.

[K] M. Kashiwara, The asymptotic behavior of a variation of polarized Hodge structure, Publ. RIMS, Kyoto Univ., $2 \mathbb{1}$ (1985), 853-875.

[K-K] M. Kashiwara, and T. Kawai, 1. The Poincaré lemma for a variation of polarized Hodge structure, preprint RIMS-540, the announcement is appeared in Proc. Japan Acad., 61, Ser. A (1985), 164-167.

[S] W. Schmid, Variation of Hodge structure; the singularities of the period mapping, Inv. Math., 22 (1973), 211-319.

[S-Z] J. Steenbrink and S. Zucker, Variation of mixed Hodge structure I, Inv. Math, 80 (1985), 485-542.

[Z] S. Zucker, Variation of mixed Hodge structure II, Inv. Math., 80 (1985), 543-565. 